\title{
Neutrophil FcyRIIA promotes IgG-mediated glomerular neutrophil capture via Abl/Src kinases
}

\author{
Hiroshi Nishi, ${ }^{1}$ Kazuhiro Furuhashi, ${ }^{1}$ Xavier Cullere, ${ }^{1}$ Gurpanna Saggu, ${ }^{1}$ Mark J. Miller, ${ }^{2}$ Yunfeng Chen, ${ }^{3}$ Florencia Rosetti, ${ }^{1}$ \\ Samantha L. Hamilton, ${ }^{2}$ Lihua Yang, ${ }^{2}$ Spencer P. Pittman, ${ }^{2}$ Jiexi Liao, ${ }^{3}$ Jan M. Herter, ${ }^{1}$ Jeffrey C. Berry, ${ }^{4}$ Daniel J. DeAngelo, ${ }^{5}$ \\ Cheng Zhu, ${ }^{3}$ George C. Tsokos, ${ }^{6}$ and Tanya N. Mayadas'

\begin{abstract}
'Department of Pathology, Brigham and Women's Hospital and Harvard Medical School, Boston, Massachusetts, USA. Division of Infectious Diseases, Department of Medicine, Washington University School of Medicine, St. Louis, Missouri, USA. ${ }^{3}$ Woodruff School of Mechanical Engineering and Institute for Bioengineering and Bioscience, Georgia Institute of Technology, Atlanta, Georgia, USA. ${ }^{4}$ Donald Danforth Plant Science Center, St. Louis, Missouri, USA. 'DDepartment of Medical Oncology, Dana-Farber Cancer Institute and Harvard Medical School, Boston, Massachusetts, USA. ${ }^{6}$ Division of Rheumatology, Department of Medicine, Beth Israel Deaconess Medical Center and Harvard Medical School, Boston, Massachusetts, USA.
\end{abstract}

\begin{abstract}
The kidney glomerular capillaries are frequent sites of immune complex deposition and subsequent neutrophil accumulation in post-infectious and rapidly progressive glomerulonephritis. However, the mechanisms of neutrophil recruitment remain enigmatic, and there is no targeted therapeutic to avert this proximal event in glomerular inflammation. The uniquely human activating Fc receptor Fc $\gamma$ RIIA promotes glomerular neutrophil accumulation and damage in anti-glomerular basement membrane-induced (anti-CBM-induced) glomerulonephritis when expressed on murine neutrophils. Here, we found that neutrophils are directly captured by immobilized IgG antibodies under physiological flow conditions in vitro through Fc $\gamma$ RIIAdependent, Abl/Src tyrosine kinase-mediated F-actin polymerization. Biophysical measurements showed that the lifetime of FcyRIIA-IgG bonds increased under mechanical force in an F-actin-dependent manner, which could enable the capture of neutrophils under physiological flow. Kidney intravital microscopy revealed that circulating neutrophils, which were similar in diameter to glomerular capillaries, abruptly arrested following anti-GBM antibody deposition via neutrophil Fc $\gamma$ RIIA and Abl/ Src kinases. Accordingly, inhibition of Abl/Src with bosutinib reduced FcyRIIA-mediated glomerular neutrophil accumulation and renal injury in experimental, crescentic anti-СBM nephritis. These data identify a pathway of neutrophil recruitment within glomerular capillaries following IgC deposition that may be targeted by bosutinib to avert glomerular injury.
\end{abstract}

\section{Introduction}

Infiltration of myeloid-derived cells in the glomerulus of the kidney is a key pathogenic event in autoantibody-mediated glomerulonephritis (GN), a leading cause of end-stage renal disease (1-4). Glomerular neutrophil accumulation occurs in Goodpasture's (or anti-glomerular basement membrane [anti-GBM]) disease, infection-related GNs, and proliferative lupus nephritis (1-3). Neutrophils are one of the earliest leukocyte subsets to be recruited to deposited autoantibodies and are known to promote glomerular injury (5). Yet, the molecular mechanisms driving immune complex-mediated (IC-mediated) neutrophil recruitment in the glomerulus, where capillaries are the major sites of leukocyte recruitment, remain poorly understood.

Low-affinity Fc $\gamma$ Rs, receptors for IgG that avidly bind ICs, are key determinants of leukocyte influx and injury following glomerular IgG deposition in models of proliferative GN. Mice deficient in the Fc $\gamma \mathrm{R} \gamma$ chain $\left(\gamma^{-/}\right)$, essential for the expression and function of all murine activating Fc $\gamma$ Rs, exhibit a marked

Authorship note: K. Furuhashi, X. Cullere, and G. Saggu contributed equally to this work. Conflict of interest: H. Nishi and T.N. Mayadas are inventors on a provisional patent, "Treatment of IgG-immune complex mediated organ damage" (serial nos. 61/990,873). Submitted: March 16, 2017; Accepted: July 18, 2017.

Reference information: / Clin Invest. 2017;127(10):3810-3826

https://doi.org/10.1172/JCI94039. reduction in leukocyte accumulation and renal injury in acute and rapidly progressive anti-GBM antibody-induced GN, and in a lupus-prone NZB/W background $(6,7)$. The low-affinity activating Fc $\gamma$ Rs on neutrophils in mice and humans structurally differ. Mice express Fc $\gamma$ RIII and Fc $\gamma$ RIV that rely on the Fc $\gamma$ R $\gamma$ chain, while humans express the uniquely human homologs Fc $\gamma$ RIIA and Fc $\gamma$ RIIIB, respectively. Fc $\gamma$ RIIA has an immunotyrosine activating motif (ITAM) in its cytoplasmic domain, and is a mediator of destructive antibody-based inflammation in autoimmunity, while Fc $\gamma$ RIIIB is a glycosylphosphatidylinositol-linked (GPI-linked) receptor whose physiological functions remain to be fully elucidated (8). Generation of mice expressing either of these human Fc $\gamma$ Rs selectively on neutrophils of $\gamma^{-/-}$mice lacking their endogenous activating Fc $\gamma$ Rs allowed us to assess the role of the human Fc $\gamma$ Rs, and specifically Fc $\gamma$ Rs on neutrophils, in disease pathogenesis (9). The surface expression level of Fc $\gamma$ RIIA on transgenic murine neutrophils and human neutrophils was comparable, while the surface expression level of Fc $\gamma$ RIIIB on transgenic neutrophils was similar to that of Fc $\gamma$ RIIA but lower than that on human neutrophils (9). Neutrophil human Fc $\gamma$ RIIA or Fc $\gamma$ RIIIB expression restored glomerular neutrophil influx in $\gamma^{-/-}$mice following crescentic anti-GBM-induced nephritis. However, only Fc $\gamma$ RIIA sustained neutrophil influx and promoted glomerular injury, suggesting that Fc $\gamma$ RIIA on neutrophils is a key molecular link 
A

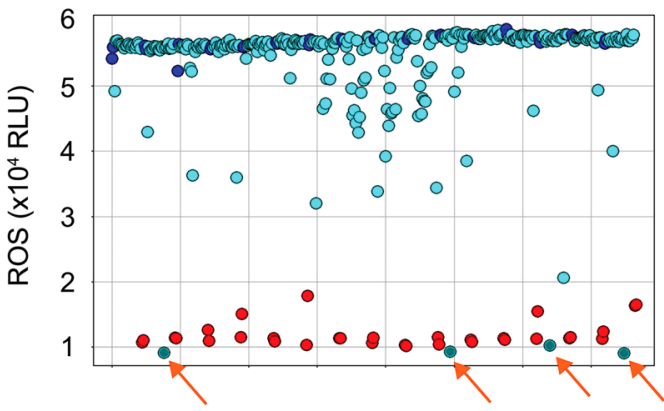

B

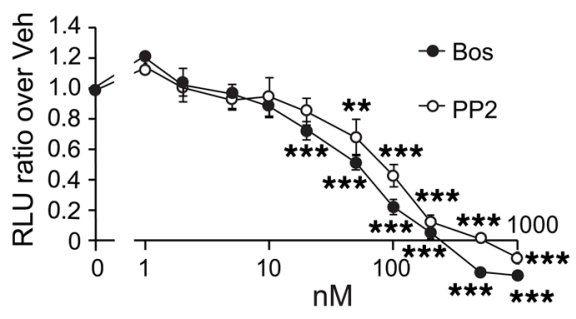

C

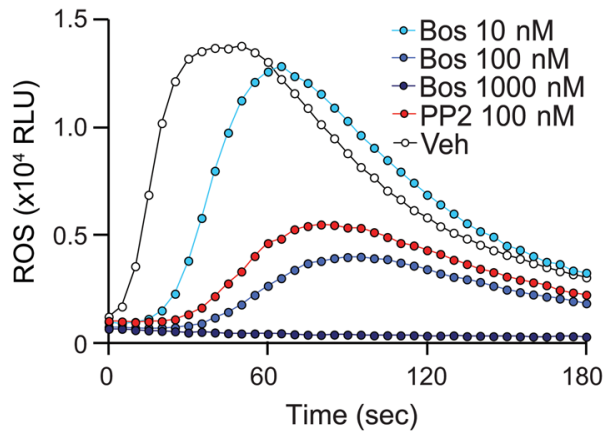

$\mathbf{E}$

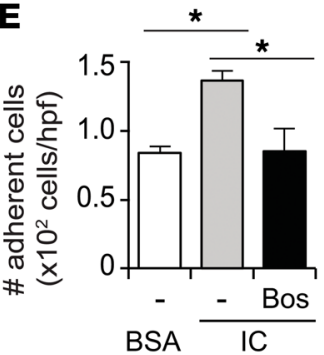

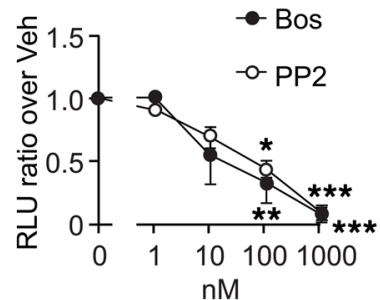

$\mathbf{F}$

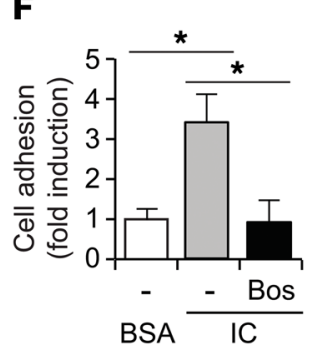

D

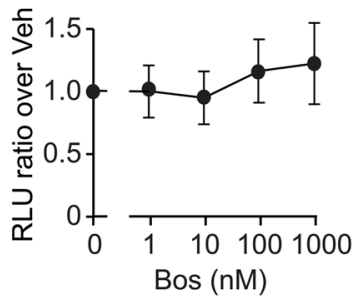

Figure 1. A small-molecule screen identifies bosutinib as the lead inhibitor of FcyRIIA functions in neutrophils. (A) Fc $\gamma R$ III ${ }^{+} / \gamma^{-1-}$ mouse bone marrow neutrophils (BMNs), suspended in fluorescent ROS probes with library compounds (light blue, at $10 \mu \mathrm{M}$ ), were loaded on BSA/anti-BSA IC-coated plates, and fluorescence was read 1 hour later. The Src inhibitor PP2 (red) and the diluent DMSO (vehicle, deep blue) were positive and negative controls, respectively. A profile with representative "hits" (arrows) is shown. (B) Bosutinib (Bos) and PP2 at indicated doses were evaluated and plotted relative to vehicle $(0 \mathrm{nM})(n=3)$. (C) BMNs were preincubated with mouse anti-human FcyRIIA and then Bos, PP2, or vehicle (Veh). ROS generation was evaluated upon addition of $F\left(a b^{\prime}\right)$, anti-mouse IgG in a luminol-based assay. A representative profile is shown (left). The average peak level of ROS was normalized to vehicle (right) $(n=3)$. (D) Bos-pretreated BMNs were stimulated with PMA, and ROS generation was assessed as in C $(n=3)$. (E) The number of adherent (left) and spread (right) cells on BSA or IC under static conditions of BMNs pretreated with vehicle $(-)$ or Bos $(n=3)$. (F) Adhesion under shear flow $\left(0.5 \mathrm{dyn} / \mathrm{cm}^{2}\right)$ to BSA- or IC-coated coverslips, of vehicle- or Bos-pretreated BMNs. The data are presented as the average fold induction normalized to the average value of the vehicle $(-) / B S A$ control $(n=3)$. Neutrophils were treated with $100 \mathrm{nM}$ Bos or $1 \mu \mathrm{M}$ PP2 unless indicated otherwise $(n=3)$. Data are mean \pm SEM.

${ }^{*} P<0.05,{ }^{* *} P<0.01,{ }^{* * *} P<0.001,1$-way ANOVA followed by Dunnett's multiple comparison test for $\mathbf{B}$ and $\mathbf{C}$ to assess dose responsiveness of the drugs compared with vehicle, and for $\mathbf{E}$ and $\mathbf{F}$.

between IC deposition, glomerular neutrophil recruitment, and end-organ damage (9). Studies in vitro suggest that neutrophils, via their own Fc $\gamma$ Rs, can be directly recruited to IgG: Fc $\gamma$ RIIIB promoted the capture of human neutrophils to plate immobilized IgG-ICs under physiological flow conditions (10, 11), while Fc $\gamma$ RIIA supported the increase in neutrophil adhesion to TNF-activated endothelial cells coated with antibodies (11). A direct interaction of Fc $\gamma$ Rs on circulating neutrophils with deposited IgG may be particularly relevant in the capillaries of the glomerulus, as IgGs deposited in the GBM are potentially accessible to circulating neutrophils via open endothelial fenestrae (12), as are anti-endothelial cell antibodies present in many glomerulonephritides (13).
Here, we explored the mechanisms driving neutrophil capture by immobilized ICs in vitro and assessed the physiological relevance of our findings by intravital microscopy and in a model of crescentic, anti-GBM nephritis. Using a large small-molecule screen on primary neutrophils, we identified the Abl/Src inhibitor bosutinib as the lead inhibitor of several Fc $\gamma$ RIIA functions and both Abl1 and Src kinases as key mediators of Fc $\gamma$ RIIA-mediated neutrophil capture on immobilized ICs under flow. Abl1 silencing or Src inhibition prevented Fc $\gamma \mathrm{R}$-mediated F-actin polymerization, which was required for IC-mediated neutrophil capture. Biophysical approaches showed that Fc $\gamma \mathrm{R}$ binding to $\operatorname{Ig} G$ was strengthened under mechanical force and required F-actin polymerization, which may enable rapid FcyR-mediated capture under flow. This 


\section{Table 1. List of top 10 biologically active compounds that significantly suppressed mouse bone marrow-derived neutrophil ROS generation induced by immobilized ICs}

$\begin{array}{lcc}\text { Compounds }(1.1 \mu \mathrm{M}) & \text { Mode of action } & \text { ROS RLU ratio } \\ \text { Bosutinib } & \text { Bcr-Abl and Src-Abl kinase inhibitor } & 0.033 \\ \text { A-443654 } & \text { Akt inhibitor } & 0.266 \\ \text { PF431396 } & \text { FAK and Pyk-2 inhibitor } & 0.301 \\ \text { CF109203X } & \text { PKC inhibitor } & 0.338 \\ \text { BX795 } & \text { PDK1 and TBK1 inhibitor } & 0.396 \\ \text { Chlorocresol } & \text { Ryanodine Ca+ receptor activator } & 0.431 \\ \text { Lck inhibitor } & \text { Lck inhibitor } & 0.462 \\ \text { Parachlorophenol } & \text { Topical antibacterial } & 0.606 \\ \text { Cresol } & \text { Antiseptic and disinfectant } & 0.761 \\ \text { Ro 31-8220 } & \text { PKC inhibitor } & 0.778\end{array}$

mode of IC-mediated neutrophil capture was evident by intravital microscopy. Endogenous mouse neutrophils rapidly arrested in glomerular capillaries in response to anti-GBM deposition in the absence of prior rolling or crawling. Simultaneous adoptive transfer of Fc $\gamma$ R-deficient and Fc $\gamma$ RIIA-expressing neutrophils or vehicle-treated and bosutinib-treated neutrophils demonstrated the importance of Fc $\gamma$ RIIA and $\mathrm{Abl} / \mathrm{Src}$ in this process, respectively. Bosutinib treatment inhibited neutrophil accumulation and renal injury in mice subjected to crescentic anti-GBM nephritis, a model of Goodpasture's syndrome (14). Thus, bosutinib, a drug currently used for the treatment of chronic myelogenous leukemia (CML) patients $(15,16)$, may be repurposed to inhibit IgG-mediated glomerular neutrophil accumulation, potentially one of the earliest steps in IgG-mediated renal inflammation.

\section{Results}

FcyRIIA-mediated neutrophil functions are inhibited by bosutinib, identified in a high-throughput screen. We established an automated, neutrophil reactive oxygen species-based (ROS-based) high-throughput drug screen in primary neutrophils for Fc $\gamma$ RIIA inhibitors using plate-bound ICs (BSA and anti-BSA) and a small-molecule library in which FDA-approved drugs are highly represented (50\%). Mature neutrophils isolated from $\gamma^{-/-}$mice showed minimal $\mathrm{H}_{2} \mathrm{O}_{2}$ generation following their incubation with plate-bound ICs, while the same expressing human Fc $\gamma$ RIIA (Fc $\gamma$ $\mathrm{RIIA}^{+} / \gamma^{-/}$) gave a robust ROS response (Supplemental Figure 1A; supplemental material available online with this article; https:// doi.org/10.1172/JCI94039DS1). Next, the ability of library compounds to inhibit Fc $\gamma \mathrm{RIIA}^{+} / \gamma^{-/-}$-induced ROS was evaluated. Vehicle control (DMSO) and PP2, an inhibitor of Src family tyrosine kinases known to signal downstream of Fc $\gamma$ Rs $(17,18)$, served as negative and positive controls, respectively. The screen of 8,483 compounds yielded 84 hits ( $1 \%$ hit rate), defined as those that have an inhibitory potential of $\geq 95 \%$ of PP2 (Figure 1A). Of the 30 compounds cherry-picked for a secondary screen (Table 1), the most potent was bosutinib, with an $\mathrm{IC}_{50}$ of 50-100 nM, which approximated that of PP2 (Figure 1B). Bosutinib is a dual Abl/Src inhibitor currently used in CML patients to inhibit Bcr-Abl, a fusion protein that leads to constitutively active Abl1 (19). Adhesion-dependent ROS generation (20), as occurs in the primary screen with platebound ICs, also relies on the CD18 integrin Mac-1 (Supplemental Figure 1B). This is consistent with previous observations that Mac1 activation by Fc $\gamma$ Rs sustains neutrophil adhesion $(21,22)$, which in our case may occur following binding of activated Mac-1 to BSA (23) in the anti-BSA/BSA ICs. To discern the effects of bosutinib on Fc $\gamma$ RIIA alone, ROS was evaluated after Fc $\gamma$ RIIA cross-linking of neutrophils in suspension; bosutinib reduced ROS generation with an $\mathrm{IC}_{50}$ of 10-50 nM (Figure 1C) while having no effect on ROS generated after PMA treatment (Figure 1D), which directly activates the NADPH oxidase (24). Fc $\gamma$ R engagement leads to the release of enzymes from granules (8) and neutrophil extracellular traps (NETs) (3, 25-27), a network of DNA fibers extruded by neutrophils. Bosutinib reduced Fc $\gamma$ RIIA-mediated release of enzymes (Supplemental Figure 1C), albeit reduced cell adhesion may also contribute to these results. Bosutinib also inhibited IC-induced NET release but not receptor-independent NETosis induced by the calcium ionophore ionomycin (Supplemental Figure 1D).

Next, the effect of bosutinib on Fc $\gamma$ R-dependent neutrophil adhesion to plate-bound ICs under static and physiological flow conditions was evaluated. Bosutinib reduced the adhesion and spreading of Fc $\gamma \mathrm{RIIA}^{+} / \gamma^{-/-}$neutrophils on ICs under static conditions (Figure 1E), which is both Fc $\gamma \mathrm{R}$ and Mac-1 dependent (21, 22). Importantly, bosutinib significantly reduced Fc $\gamma \mathrm{RIIA}{ }^{+} / \gamma^{-/-}$ neutrophil tethering to immobilized ICs under physiological flow (Figure $1 \mathrm{~F}$ ), which is strictly $\mathrm{Fc} \gamma \mathrm{R}$ dependent $(10,11)$ and may serve as a surrogate for neutrophil accumulation following intravascular IC deposition. Similarly, human neutrophils treated ex vivo with bosutinib exhibited a significant reduction in Fc $\gamma$ RIIA-mediated (but not PMA-mediated) ROS generation ( $\mathrm{IC}_{50}$ of 1-5 nM) (Figure 2, A and B), release of enzymes (Supplemental Figure 1E), adhesion and spreading on ICs under static conditions (Figure 2C), and neutrophil capture on plate-bound ICs under flow (Figure 2D). Likewise, the Abl inhibitors imatinib and nilotinib, which, unlike bosutinib, do not affect Src activity (28), reduced human neutrophil Fc $\gamma$ RIIA-mediated ROS generation (Figure 2B) and adhesion to ICs under static (Figure 2C) and flow (Figure 2D) conditions. This suggests that Abl proteins play a key role in these Fc $\gamma$ R functions. The Src kinase inhibitor PP2 also reduced ROS generation (Figure 2A) and neutrophil adhesion to ICs under static conditions (Figure 2C) as expected (18). The aforementioned studies evaluating the capture of human neutrophils on immobilized ICs were largely dependent on Fc $\gamma$ RIIIB $(10,11)$, which is GPI-linked but still signals via nonreceptor tyrosine kinase (29). To interrogate the effects of bosutinib specifically on Fc $\gamma$ RIIA-mediated capture in human neutrophils, we evaluated neutrophil adhesion to TNF-activated human dermal microvascular endothelial cells (HDMECs) coated with ICs in situ, which results in an increase in neutrophil adhesion compared with TNF alone that is Fc $\gamma$ RIIA and not Fc $\gamma$ RIIIB dependent (11). As reported (11), ICs increased neutrophil adhesion to TNF-activated endothelial cells, which was abrogated by functional blocking anti-Fc $\gamma$ RIIA but not anti-Fc $\gamma$ RIIIB antibody (Figure 2E). Importantly, human neutrophil pretreatment with bosutinib or imatinib markedly reduced neutrophil adhesion on TNF-activated/IC-coated HDMECs to levels observed for endothelial cells treated with TNF alone (Figure 2E). 
A
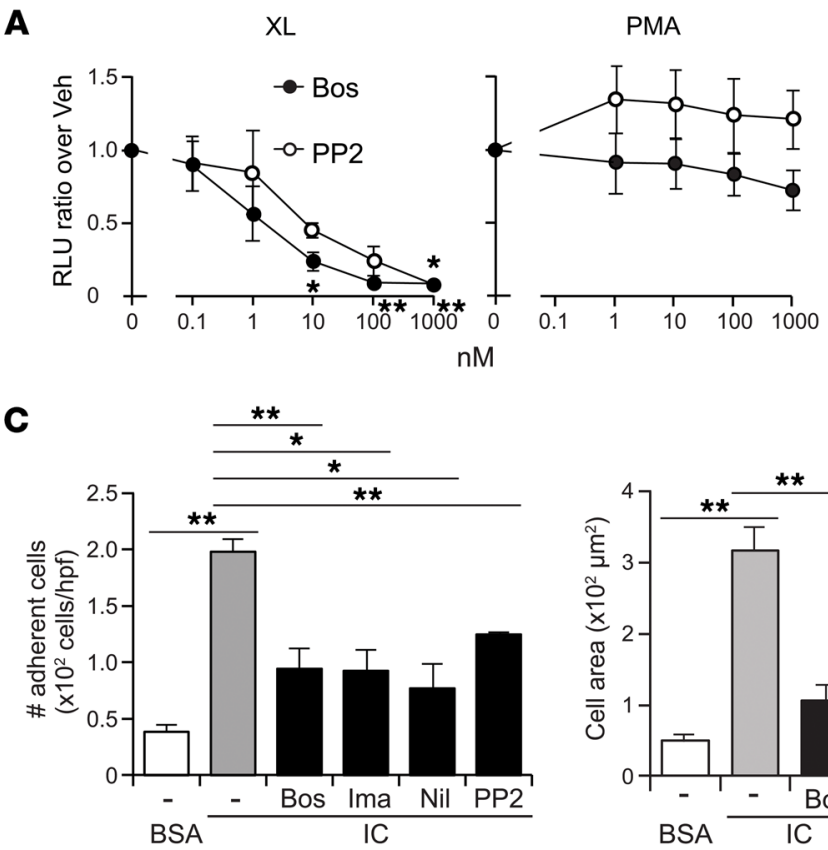

D

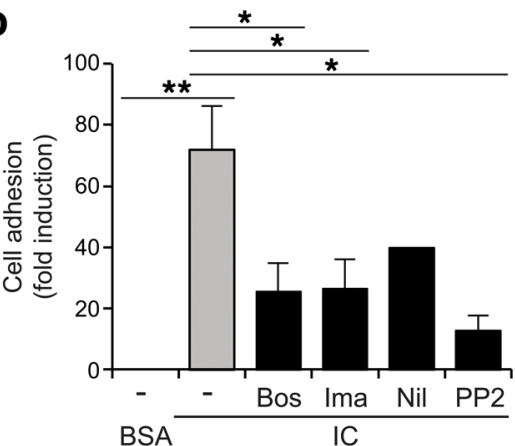

$\mathbf{E}$
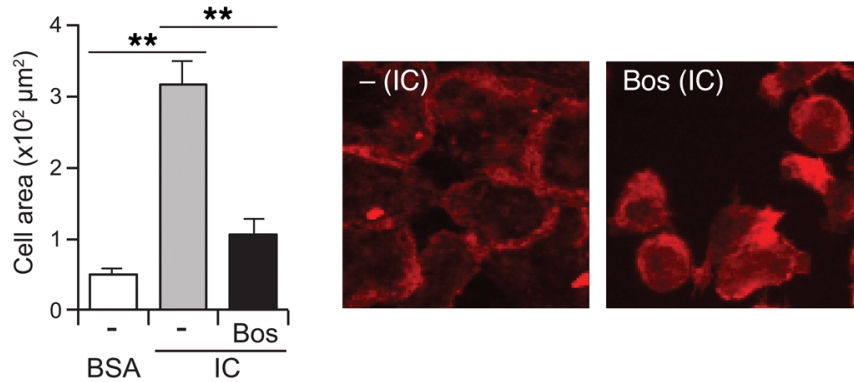
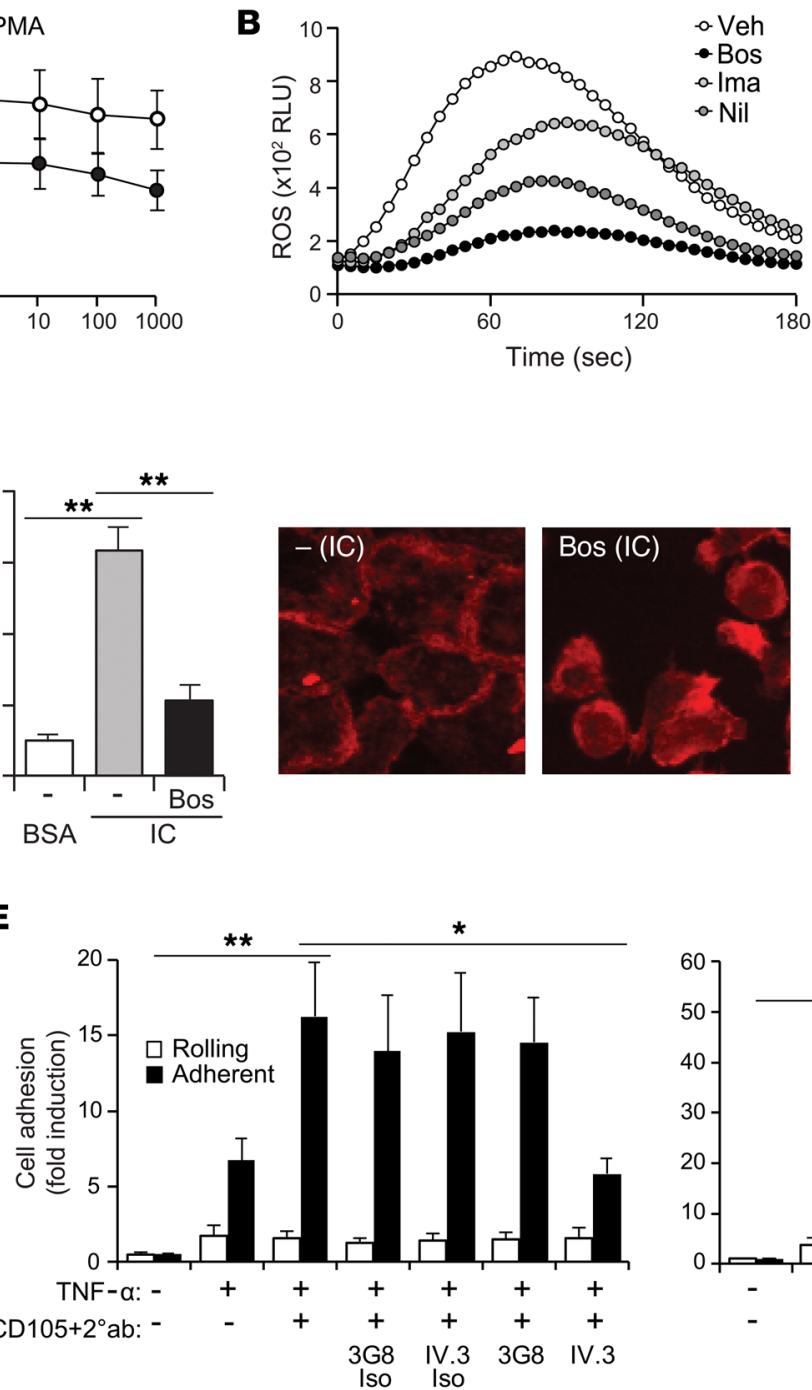
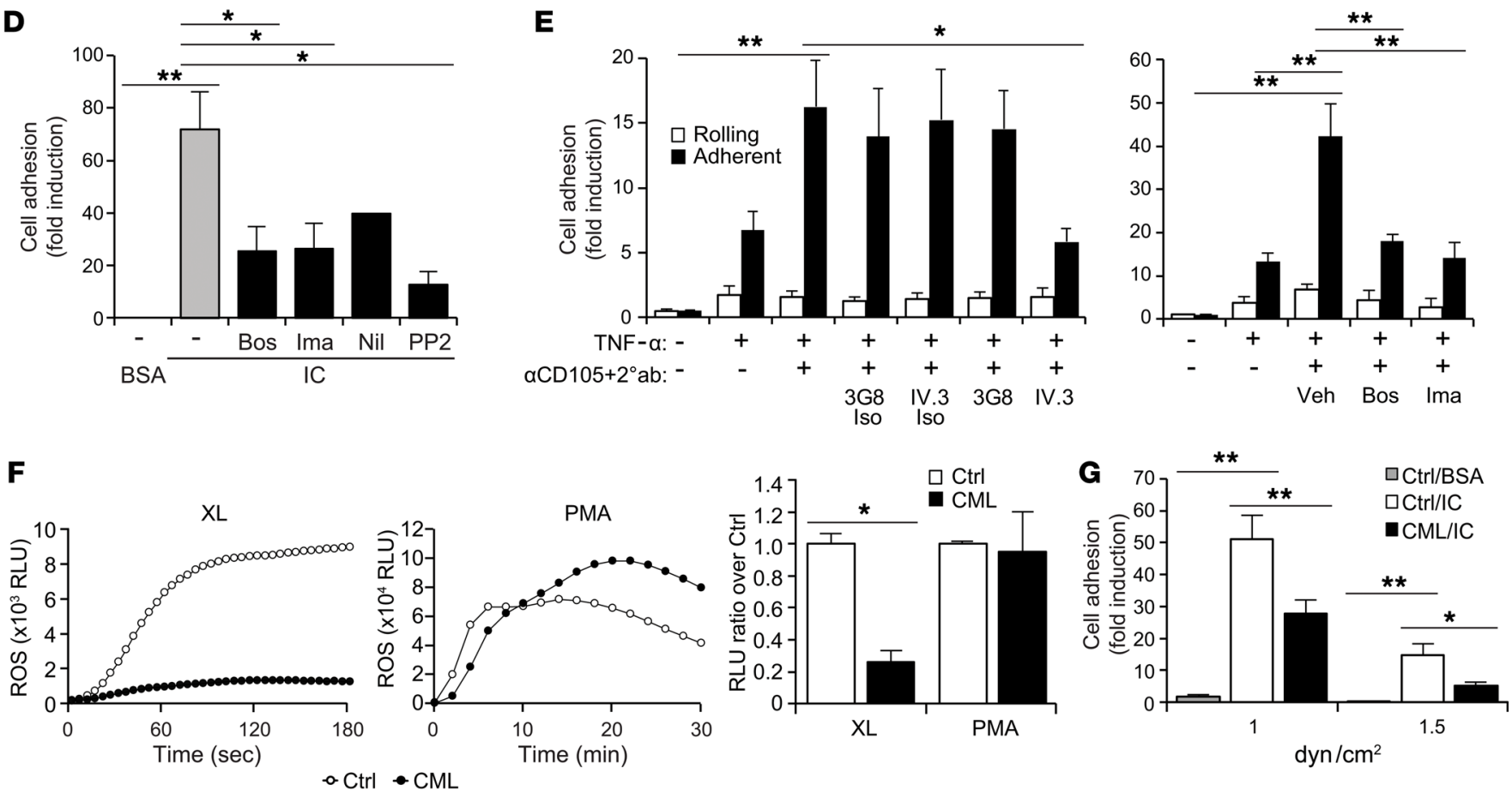

Figure 2. Bosutinib inhibits FcyRIIA functions in human neutrophils. (A) ROS after Fc $\gamma R$ IIIA cross-linking (XL) (left) or PMA (right) in bosutinib-pretreated (Bos) and PP2-pretreated neutrophils as in Figure 1, C and D $(n=3)$. (B) Representative profile of XL-induced ROS after treatment with vehicle or $100 \mathrm{nM} \mathrm{Bos,} \mathrm{imatinib} \mathrm{(Ima),} \mathrm{or} \mathrm{nilotinib} \mathrm{(Nil)}(n=3)$. (C) Neutrophils pretreated with $100 \mathrm{nM}$ of the indicated compounds, $1 \mu \mathrm{M}$ PP2, or vehicle (DMSO, -) on BSA- or IC-coated plates, stained with fluorescent phalloidin, and quantitated for adherent cells (left) and spread cell area (right) $(n=3)$. Representative images are shown. Original magnification, $\times 600$. (D) The number of adherent cells pretreated as in $\mathbf{C}$ and drawn across BSA- or IC-coated plates at $1.0 \mathrm{dyn} / \mathrm{cm}^{2}$ was calculated and is presented as the fold induction compared with vehicle (-)/BSA control ( $n=3$, except for Nil, $n=2)$. (E) HDMECs were treated without (-) or with $(+)$ TNF and/or anti-CD105 plus a secondary antibody $\left(2^{\circ}\right.$ ab). Neutrophils pretreated with anti-Fc $\mathrm{RIIIB}$ (3G8), Fc $\gamma$ RIIA (IV.3), or isotype (Iso) controls (left panel), or with $100 \mathrm{nM}$ Bos or Ima (right panel), were drawn across HDMECs at $1.5 \mathrm{dyn} / \mathrm{cm}^{2}$. The number of rolling and adherent cells was assessed. Fold induction was relative to untreated HDMECs $(n=3)$. $(\mathbf{F}) \mathrm{XL}-\mathrm{or}$ PMA-induced ROS in blood leukocytes from 3 CML patients receiving Bos and matched normal volunteers (Ctrl). A representative ROS profile (left panel) and average fold induction relative to the XL (Ctrl) sample (right panel) are shown $(n=3)$. (G) CML and control leukocytes were analyzed as in D at 1.0 and $1.5 \mathrm{dyn} / \mathrm{cm}^{2}$. Fold induction is relative to the control sample on BSA (Ctrl/BSA) $(n=3)$. All data are expressed as the mean \pm SEM. ${ }^{*} P<0.05,{ }^{* *} P<0.01,1$-way ANOVA followed by Dunnett's multiple comparisons test for $\mathbf{A}$ to assess dose responsiveness of the drugs compared with vehicle and also for $\mathbf{C}-\mathbf{E}$ and $\mathbf{G}$, and by 2 -tailed unpaired $t$ test in $\mathbf{F}$. 
A

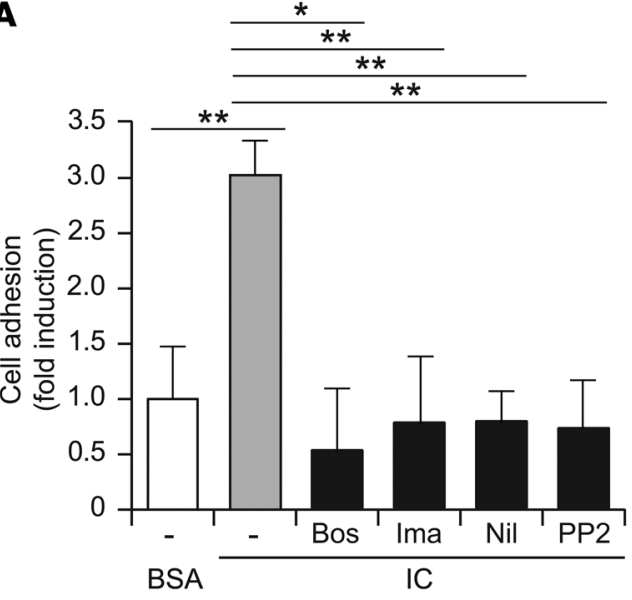

D

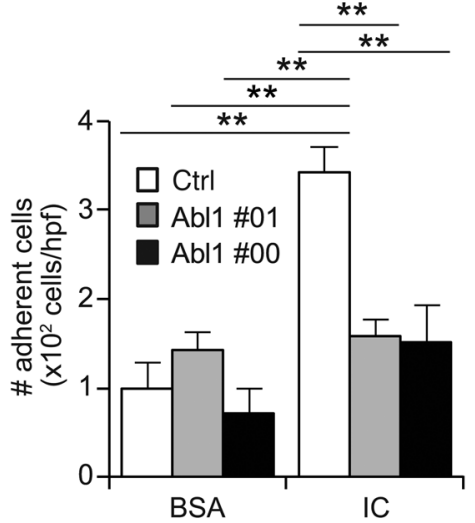

B

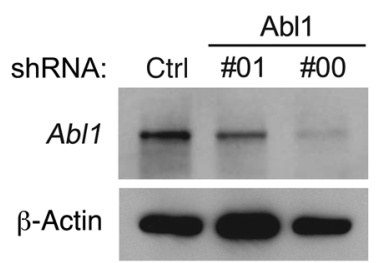

C
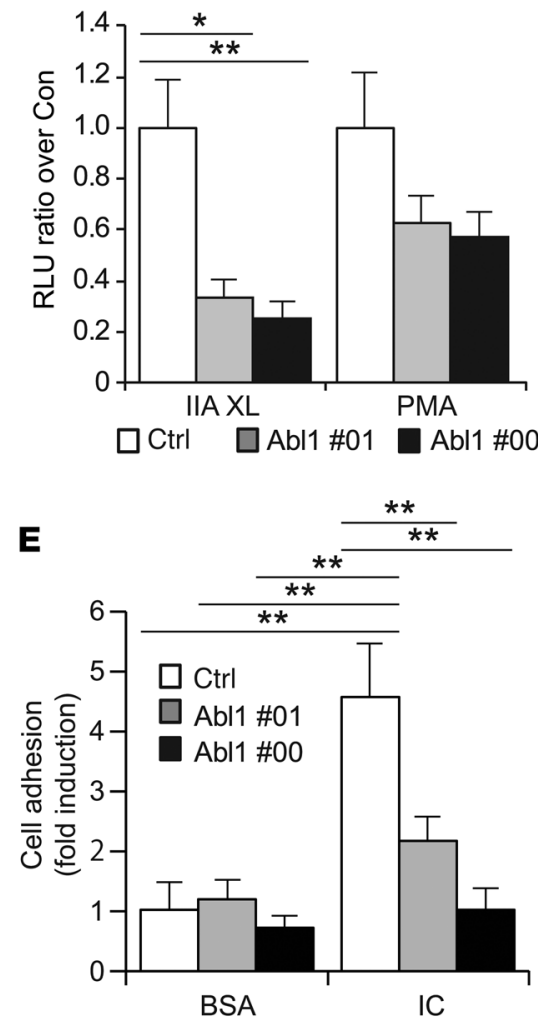

E

F

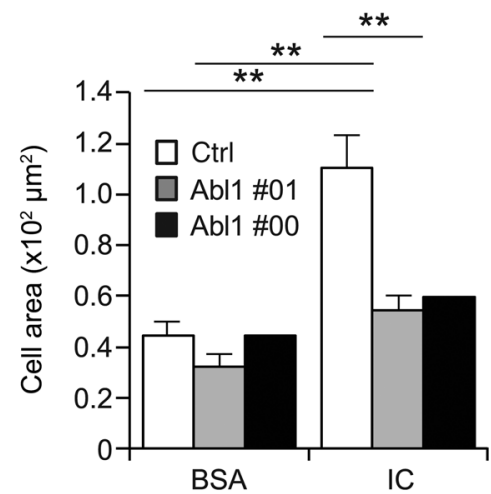

G

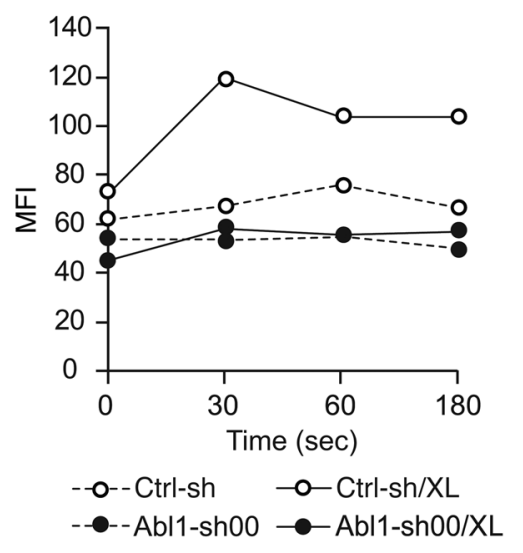

H

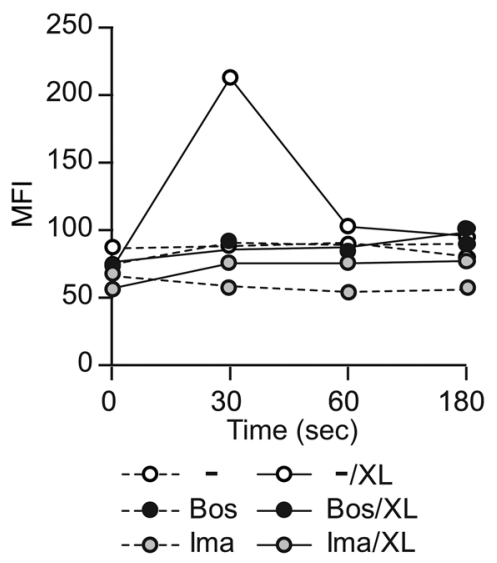

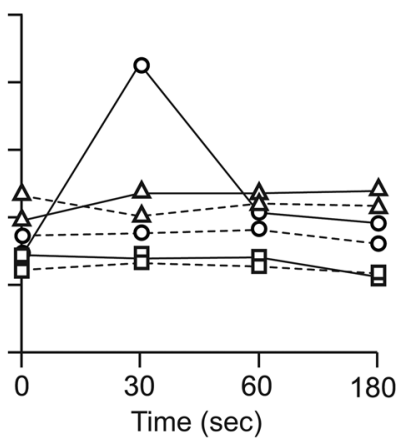

- - - - - - - - $/ X L$

--口-- cytD - - - cytD /XL

- $\Delta$ - PP2 $-\Delta-$ PP2/XL 
Figure 3. Role for Abl kinases in FcyRIIA-mediated neutrophil functions. (A) Differentiated HL-60 cells pretreated with vehicle (-), bosutinib (Bos), imatinib (Ima), nilotinib (Nil), or PP2 adherent to ICs or BSA under flow. Fold induction is relative to vehicle $(-)$ on BSA $(n=3)$. (B) A representative Western blot of cells expressing Abl1 (Abl1 \#01 and \#00) or control shRNA and analyzed for Abl or $\beta$-actin (loading control) $(n=3)$. (C) ROS in cells in B subjected to Fc $\gamma$ RIIA cross-linking (XL) or treated with PMA. The peak level was normalized to control shRNA cells $(n=3)$. (D) Cells on BSA- or IC-coated plates were stained for actin, and the number of adherent cells (left) and the area of a single spread cell (right) were calculated ( $n=3$, except in the right panel, $n=2$ for treatment with shRNA \#00). (E) Cell adhesion to BSA or IC at $0.35 \mathrm{dyn} / \mathrm{cm}^{2}$. Fold induction is relative to control cells on BSA $(n=3)$. (F) Western blot analysis of indicated proteins with or without Fc $\gamma$ RIIA XL for times in seconds. $\beta$-Actin is the loading control. One representative of 3 is shown. (G and $\mathbf{H}$ ) F-actin polymerization analyzed by flow cytometry (mean fluorescence intensity, MFI) at indicated times after XL and staining with NBD-phallacidin. Omission of the primary antibody is the negative control. Shown is 1 representative of 3 experiments for Abl1or control shRNA-expressing cells (C), and for cells treated with vehicle $(-)$ or indicated inhibitors before $\mathrm{XL}(\mathbf{H})$. For $\mathbf{A}-\mathbf{H}$, cells were treated with 1 $\mu \mathrm{M}$ of Bcr-Abl inhibitors or PP2, or $3 \mu \mathrm{M}$ cytochalasin D (cytD). All data are mean \pm SEM. ${ }^{*} P<0.05,{ }^{*} P<0.01$, 1-way ANOVA followed by Dunnett's multiple comparison test for $\mathbf{A}$ and $\mathbf{C}-\mathbf{E}$.

Thus, Abl/Src inhibition reduced Fc $\gamma$ RIIA-mediated adhesion to ICs while sparing the classical adhesion cascade associated with TNF activation of the endothelium (30).

To determine whether bosutinib reduces Fc $\gamma$ R-mediated activity when administered in vivo, $\mathrm{Fc} \gamma \mathrm{R}$ functions were evaluated in neutrophils from CML patients receiving bosutinib. Total leukocytes in peripheral blood of CML patients and matched normal volunteers were evaluated immediately after red blood cell (rbc) lysis, as bosutinib's effect on neutrophils is reversible over time (data not shown). The percentage of neutrophils and neutrophil Fc $\gamma$ RIIA levels on the surface were similar between samples (Supplemental Figure 2, A and B). An 80\% reduction in Fc $\gamma$ RIIA-mediated ROS generation was observed in patients compared with normal volunteers, while PMA-induced ROS was similar in both groups (Figure $2 \mathrm{~F}$ ). The interaction of neutrophils from bosutinib-treated patients with immobilized ICs under flow was significantly reduced compared with the interaction of those from the normal donors (Figure $2 \mathrm{G})$. Neutrophils obtained from newly diagnosed, untreated CML patients exhibited a partial reduction in Fc $\gamma$ RIIA-induced ROS generation compared with those from normal volunteers, albeit this was not significant (peak RLU: normal, $1.00 \pm 0.83$; CML patients, $0.51 \pm 0.43 ; n=3$ per group, $P=0.63$ ).

FcyRIIA activation is initiated by ITAM phosphorylation, which triggers the docking of Syk and its phosphorylation by Src kinases that is followed by several downstream signals, including the phosphorylation and subsequent activation of the Rho GTPase guanine exchange factor Vav; Pyk-2 downstream of Syk; PI3K/Akt; MAPK/ ERK; and NADPH oxidase components such as $\mathrm{p} 40^{\text {phox }}(17,18)$. Bosutinib inhibited Fc $\gamma$ RIIA cross-linking-induced phosphorylation of these components as well as Crkl, a known Abl substrate (31) in Fc $\gamma$ RIIA $/ \gamma^{-1-}$ neutrophils (Supplemental Figure 3A). Similar results were obtained with human neutrophils (Supplemental Figure 3B).

Abl1 regulates F-actin polymerization needed for neutrophil FcyRIIA-mediated recruitment and force-induced enhancement of Fc $\gamma R$ $I A$ interaction with $I g G$. To further interrogate Abl1's contribution to Fc $\gamma$ RIIA functions, we used differentiated human HL-60 cells, which are genetically tractable neutrophil-like cells that express Fc $\gamma$ RIIA but have no detectable Fc $\gamma$ RIIIB and low expression of CD18 integrins (ref. 11 and data not shown). Treatment with bosutinib or other Bcr-Abl inhibitors significantly inhibited HL-60 cell interaction with ICs under flow (Figure 3A) as demonstrated for human neutrophils (Figure 2D). HL-60 cells were transduced with Abl1 shRNAs to 2 independent sequences that stably reduced Abl1 protein compared with the shRNA control (Figure 3B). Both Abl1 shRNAs significantly attenuated Fc $\gamma$ RIIA-mediated ROS generation compared with control shRNA (Figure 3C), while PMA-induced ROS was only partially affected (Figure 3C). Abl1 silencing reduced cell adhesion and spreading on ICs under static conditions (Figure 3D), and markedly inhibited adhesion to ICs under flow (Figure 3E). Abl1-silenced cells exhibited a decrease in FcyRIIA-induced phosphorylation of Crkl but not Pyk-2, Vav, ERK1/2, and Akt (Figure 3F), suggesting that Abl1 was not essential for proximal Fc $\gamma \mathrm{R}$-mediated signaling events. Thus, the reductions in signaling observed with bosutinib in human neutrophils (Supplemental Figure 3A) likely reflect its known targeting of Src and the STE20 serine/threonine kinases $(28,32)$.

Among tyrosine kinases, Abl kinases (Abl1 and the Abelson-related gene, Arg) are unique in being able to directly bind and regulate the polymerization of F-actin (33). Fc $\gamma$ RIIA cross-linking increased actin polymerization within seconds in control shRNA HL-60 cells as assessed by FACS analysis of NBD-phallacidinstained cells (34). The maximal change in fluorescence intensity, which reflects actin polymerization (Figure 3G), was completely prevented in Abl1-silenced cells (Figure 3G) or following treatment with Bcr-Abl inhibitors (Figure 3H). The Src inhibitor PP2 similarly inhibited F-actin polymerization (Figure $3 \mathrm{H}$ ). F-actin polymerization was required for Fc $\gamma$ RIIA-mediated ROS generation and neutrophil capture by ICs under flow, with a greater requirement for F-actin in the latter. That is, treatment with cytochalasin D, an inhibitor of F-actin polymerization, partially reduced Fc $\gamma$ RIIA-induced ROS generation (RLU at peak: vehicle, 1,879 $\pm 971 ; 20 \mu \mathrm{M}$ cytochalasin D, 1,037 \pm 483 ), while it markedly inhibited HL-60 tethering to plate-immobilized ICs (data not shown) as well as human neutrophil interaction with immobilized ICs (Figure 4A) or IC-coated HDMECs (Figure 4B) under flow. However, cytochalasin D had no affect on neutrophil adhesion to ICs under static conditions (Figure 4C). Thus, polymerized actin was not required when the on-rate of Fc $\gamma \mathrm{R}-\mathrm{IgG}$ binding was not limiting, as is the case under static conditions, while it was essential under shear flow, which is governed by the force-dependent off-rate of the Fc $\gamma$ R-IgG interactions. This, together with the observed ability of Fc $\gamma \mathrm{R}$ to bind ICs under flow, prompted us to determine whether mechanical force increases Fc $\gamma$ RIIA-IgG bond lifetimes, referred to as catch bonds (35), which we reasoned would facilitate capture of neutrophils under flow and may require F-actin polymerization. For this, Jurkat cells lacking CD18 integrins (36) were engineered to express Fc $\gamma$ RIIA to evaluate the role specifically of this Fc $\gamma \mathrm{R}$, in the absence of Fc $\gamma$ RIIIB, in the aforementioned cell responses. Fc $\gamma$ RIIA-expressing Jurkat cells bound ICs under flow, and cytochalasin D reduced this binding (Figure 4D) without affecting cell binding to ICs under static conditions (Figure 4E). Importantly, using a biomembrane force probe, we demonstrated that Fc $\gamma$ RIIA formed catch bonds with $\operatorname{IgG}$ at forces ranging from 5 to $25 \mathrm{pN}$, 
A

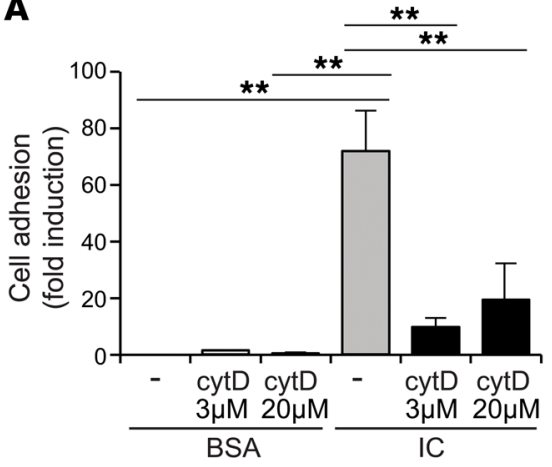

B

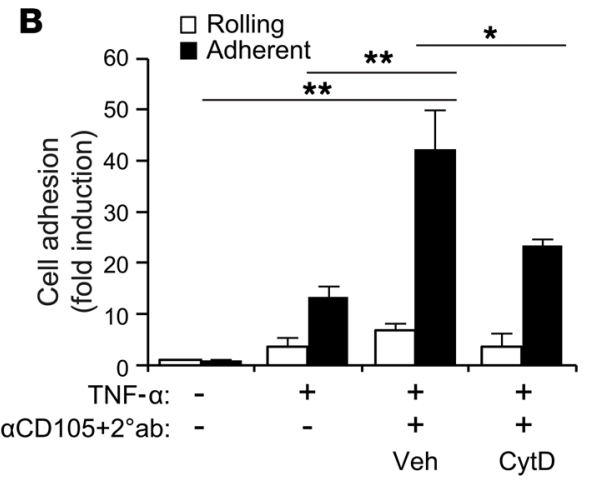

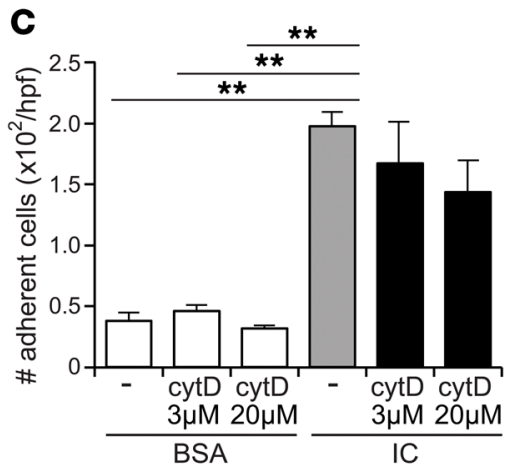

D

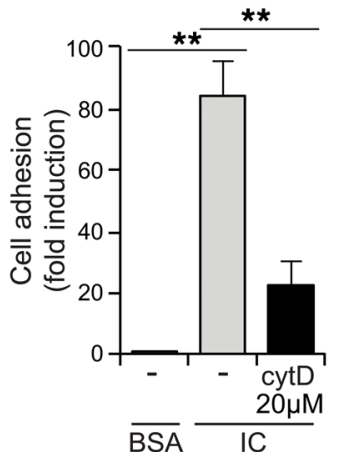

E

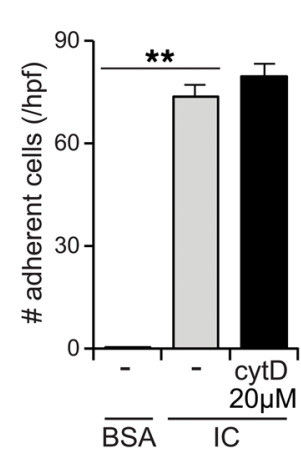

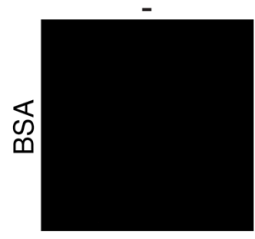

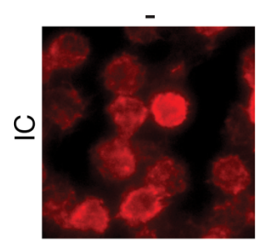

cytD

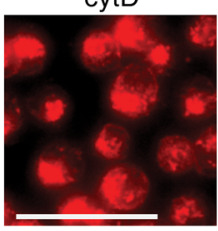

$\mathbf{F}$

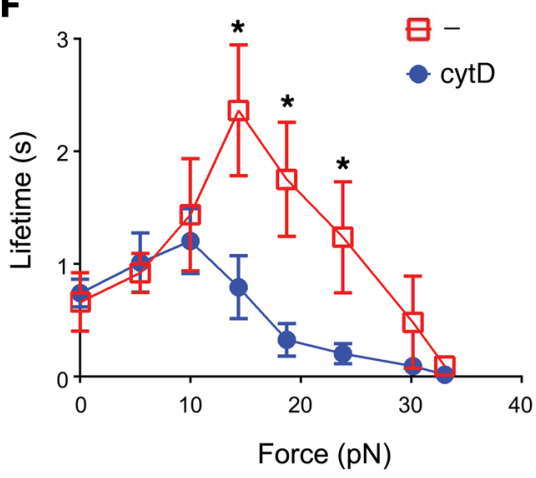

Figure 4. Actin polymerization is required for force-induced FcyRIIA-mediated interaction with IgC. (A) Human peripheral blood neutrophils were treated with 2 different doses of cytochalasin D (cytD) or with DMSO (-), and adhesion of cells to an IC- or BSA-coated surface under flow (1.0 dyn/cm²) was assessed. Fold induction is relative to vehicle-treated $(-)$ samples on ICs $(n=3)$. (B) Human peripheral blood neutrophils were pretreated with cytD and drawn across HDMECs treated with TNF and anti-endoglin as indicated and detailed in Figure 2E. Fold induction is relative to untreated HDMECs $(n=3)$. (C) Human neutrophils were treated with 2 different doses of cytD or vehicle (DMSO, -), and the number of cells adherent to IC- or BSA-coated surfaces under static conditions was assessed $(n=3)$. ( $(\mathbf{D}$ and $\mathbf{E})$ Jurkat cells expressing Fc $\gamma$ RIIA but mutagenized to lack CD18 integrins were evaluated for adhesion to ICs or BSA under flow (D) or static (E) conditions in the presence or absence of cytD $(n=3)$. Representative images for the static assay are shown to illustrate changes in actin distribution in cytD-treated versus vehicle-treated (-) neutrophils adherent to ICs. Scale bar: $25 \mu \mathrm{m}$. (F) Force-dependent lifetimes of single bonds (in seconds) between hlgG1-immobilized beads and Fc $\gamma R$ IIIA-transfected Jurkat cells in the presence (blue circles) or absence (-, red squares) of cytD. Each point represents more than 30 repeated measurements. Data are expressed as mean $\pm \mathrm{SEM} .{ }^{*} P<0.05$, ${ }^{* *} P<0.01,1$-way ANOVA followed by Dunnett's multiple comparison test for $\mathbf{A}-\mathbf{E}$ and 2-tailed unpaired $t$ test for $\mathbf{F}$.

while at higher forces the catch bonds transitioned to slip bonds. Cytochalasin D treatment had no significant effect on bond lifetimes at $0-8 \mathrm{pN}$ force but markedly inhibited bond stabilization at higher mechanical forces (Figure $4 \mathrm{~F}$ ). Thus, Fc $\gamma$ RIIA forms catch bonds with IgG, and this relies on F-actin polymerization.

Together, these data demonstrate that Fc $\gamma$ RIIA induces F-actin polymerization via Abl1 and Src kinases and that F-actin polymerization supports force-induced Fc $\gamma$ RIIA interactions with IgG, which may facilitate neutrophil capture by IgG under flow.

Rapid neutrophil capture within glomerular capillaries following anti-GBM antibody. We exploited 2-photon intravital microscopy to examine neutrophil behavior in the kidney under steady-state conditions and 1.5 hours after administration of rabbit nephrotoxic antisera, which leads to IgG deposition on the endothelium and GBM and will herein be referred to as anti-GBM antibody. We used LysM-GFP mice to evaluate endogenous neutrophils, focusing on early neutrophil-vessel wall interactions. Notably, neutrophils are readily distinguishable from monocytes and macrophages, as they are 5-10 times brighter than $\mathrm{CD}_{11 b^{+}}$, Ly6 $6 \mathrm{G}^{-}$monocytes with the brightest $\mathrm{GFP}^{+}$ cells (>2 logs over background) being preferentially detected with our imaging settings. These cells are more than 95\% neutrophils based on flow cytometric analysis $(37,38)$ and intravital microscopy analysis of LysM-GFP mice injected with a neutrophil-specific anti-Ly6G antibody (1A8) (Supplemental Figure 4, $\mathrm{A}-\mathrm{C})$. The right kidney was surgically exteriorized and imaged (39), and tissue perfusion was verified by bright-field observation of robust capillary flow and by injection of Q-dots into the retro-orbital sinus. In both control and anti-GBM-treated mice, neutrophils were visible passing through and arresting transiently ("fluttering") in glomerular capillaries, which we show approximated the diameter of a neutrophil (Figure 5, A and B). This "fluttering" behavior was also observed with small beads injected i.v. (data not shown), suggesting that hemodynamic flow variations in the tortuous glomerular capillary bed account for this behavior. Time-lapse recordings (13 frames per second) were taken for up to 20 minutes, and the number of arrested neutrophils at $t=0$ and $t=9$ minutes was counted in both groups. In control animals, a few arrested neutrophils were present in glomeruli, but in anti-GBM-treated mice, these numbers increased significantly (Figure 5C). Neutrophil duration time was plotted for each group, and both distributions were clear- 
A
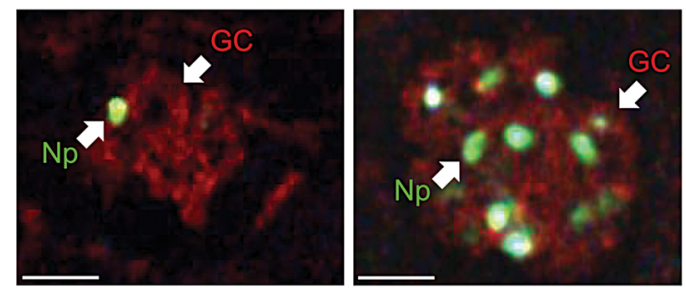

C

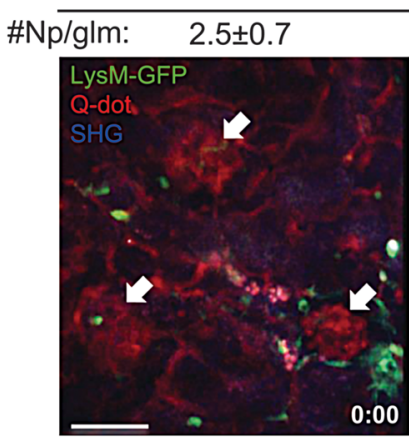

Ctrl

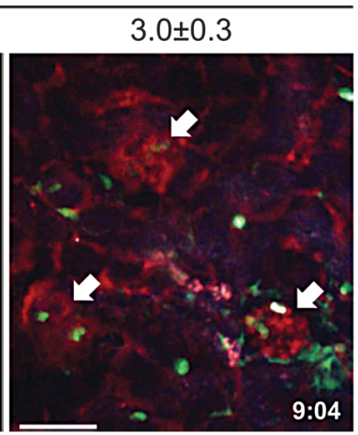

D

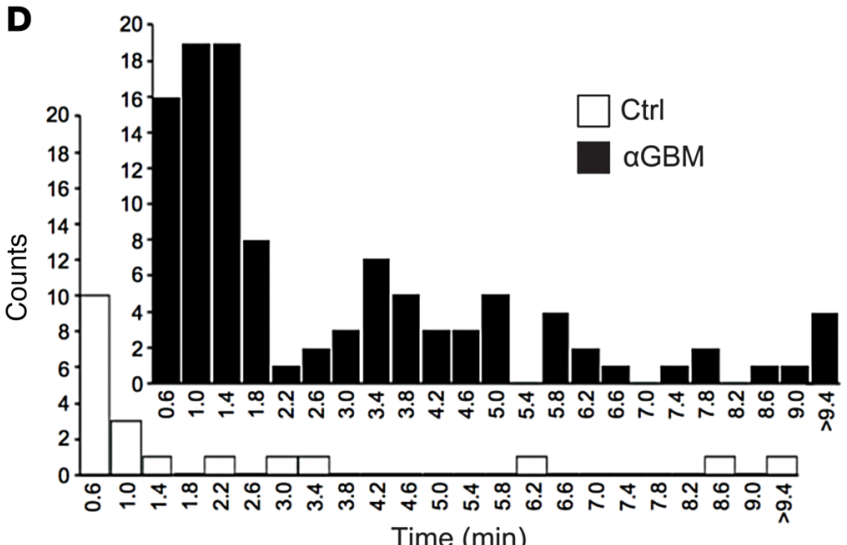

B

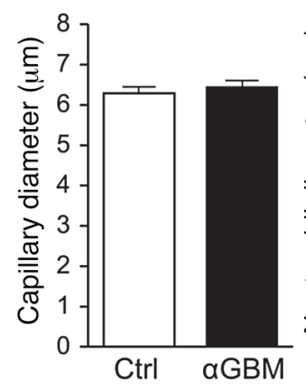

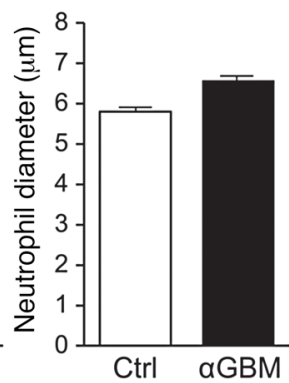

$\alpha$ GBM

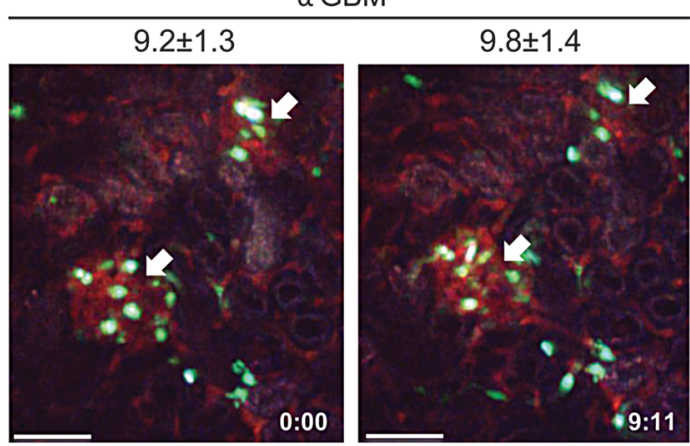

E

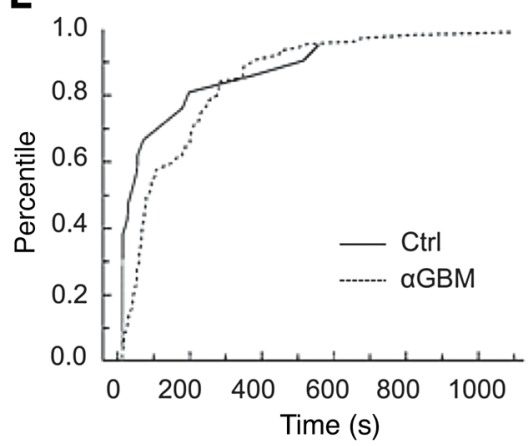

$\mathbf{F}$

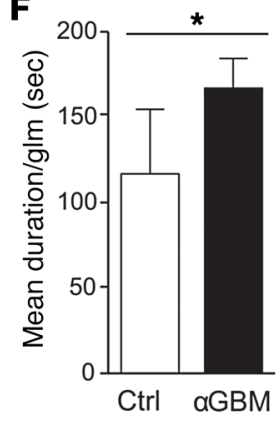

Figure 5. In vivo neutrophil trafficking in glomerular capillaries at steady state and after anti-GBM treatment in LysM-GFP mice. In vivo 2-photon timelapse imaging of glomeruli in untreated LysM-GFP mice at steady state (untreated, control) and 1.5-2 hours after anti-GBM ( $\alpha$ GBM) treatment ( $n=3$ mice, 5 glomeruli for control; $n=4$ mice, 6 glomeruli for $\alpha G B M$ ). Imaging parameters were set to preferentially visualize GFP ${ }^{+}$neutrophils (see Supplemental Figure 4A). (A and B) Glomerular capillaries (GC, red, Q-dot 565) and neutrophils (Np, green, LysM-GFP) in a control (left) and $\alpha$ GBM-treated (right) mouse are shown (A). Capillary lumen and neutrophil diameters were calculated and found to be similar in size with no significant differences between control and $\alpha \mathrm{CBM}$ groups (B) by a 2-tailed unpaired $t$ test. (C) The mean \pm SEM of neutrophils per glomerulus (glm) (arrows) in untreated and $\alpha$ GBM-treated LysM-GFP mice were counted at 0 and 9 minutes, and representative images are shown. SHG, second-harmonic generation. Scale bars: $25 \mu \mathrm{m}$ (A), $50 \mu \mathrm{m}$ (C). (D) Distributions of glomerular duration times for control and $\alpha$ GBM groups plotted in minutes. Duration times were clearly non-normal, and control and $\alpha \mathrm{CBM}$ groups were significantly different using a 2-tailed Mann-Whitney $U$ test, $P<0.01$. (E) Distributions using a 2-sample Kolmogorov-Smirnov (KS) test comparison percentile plot. The group duration time distributions were significantly different using a $\mathrm{KS}$ test, $P<0.01, D=0.3813$. (F) A bar graph of mean $\pm \mathrm{SEM}$ is shown to provide a sense of scale for the dwell times. ${ }^{*} P<0.01$ by 2 -tailed unpaired $t$ test.

ly non-normally distributed with many short duration events. Despite the qualitative similarities, neutrophils in the anti-GBM group had significantly increased duration times compared with those in the control group (2-tailed Mann-Whitney $U, P<0.01$ ) (Figure 5D). We also compared the shape of the duration time distributions using a 2-sample Kolmogorov-Smirnov (KS) test (Figure 5E). The KS test comparison percentile plot showed that the duration time distributions were significantly different $(P<$ $0.01, D=0.3813$ ), with the plots diverging quickly at short durations (less than 60 seconds) and the anti-GBM group having an increased proportion of adhesion events in this time period. The mean duration time increased in the anti-GBM group compared with control (Figure 5F), as did the median value (control, 36.09 \pm 37.90 seconds; anti-GBM, $80.73 \pm 17.40$ seconds). The "stop and go" behavior of neutrophils in the glomerular capillaries (Supplemental Videos 1 and 2) was strikingly different from the slow neutrophil rolling and intraluminal crawling observed in larger vessels $(20-30 \mu \mathrm{m})$ in other organs and inflammatory settings $(37,38)$. Indeed, neutrophil crawling was observed infrequently, with rates of $4.29 \pm 1.1$ and $7.2 \pm 2.24$ cells per glomer- 
A

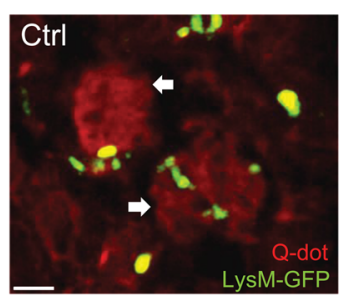

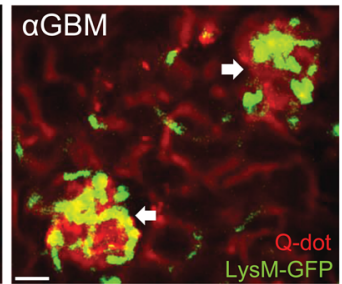

B

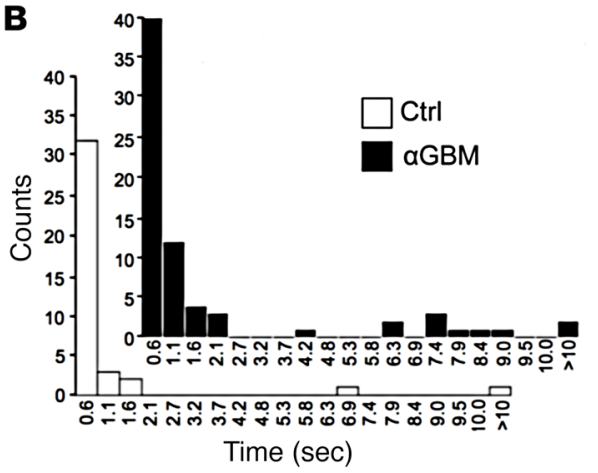

C

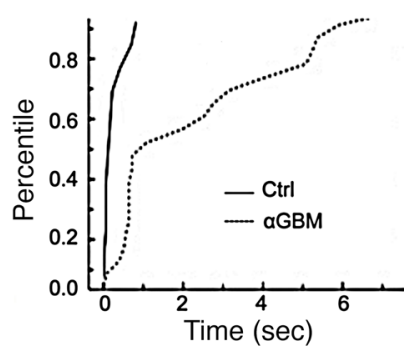

D

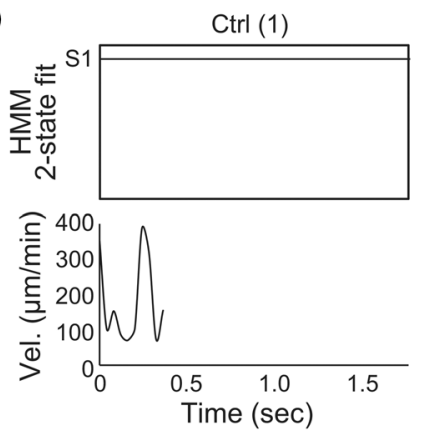

E

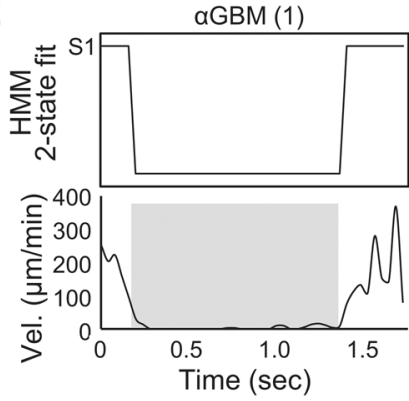

Ctrl (2)

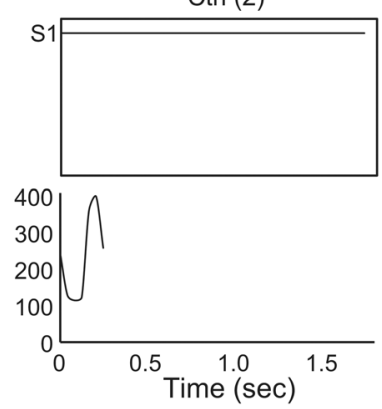

aGBM (2)

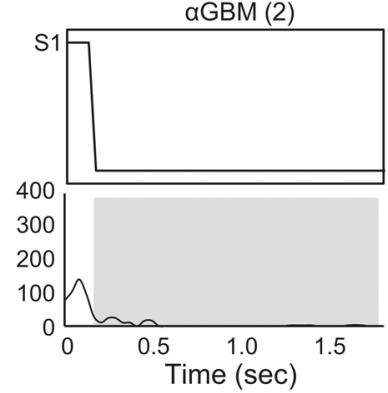

$\mathbf{F}$

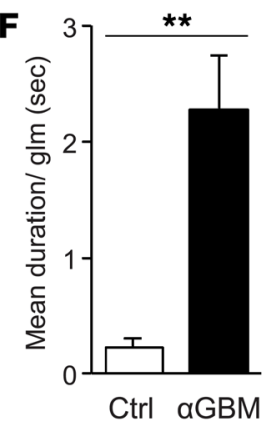

H

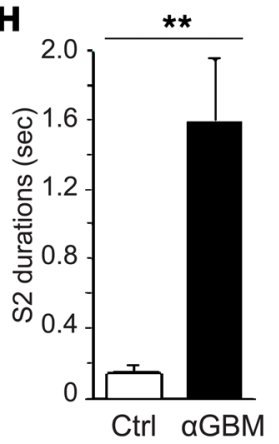

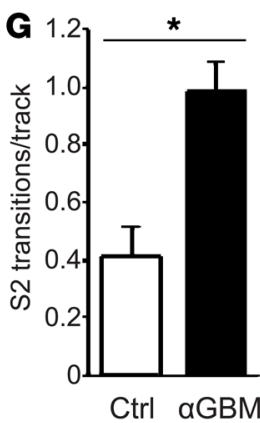

I

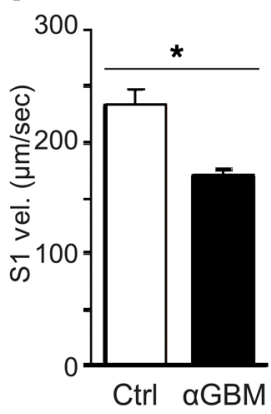

Figure 6. Analysis of short-duration neutrophil-vessel wall interactions in control and anti-GBM-treated LysM-GFP mice. In vivo 2-photon video-rate imaging of glomeruli in untreated LysM-GFP mice (Ctrl) ( $n=3$ mice, 5 glomeruli) and in LysM-GFP mice 1.5-2 hours after anti-GBM ( $\alpha$ CBM) treatment ( $n=3$ mice, 4 glomeruli). (A) A video-rate image of steady-state neutrophil (green) trafficking behavior in glomerular capillaries (red, Q-dot 565) (white arrows) of a control mouse (left panel) and a mouse after $\alpha$ CBM treatment (right panel). Images are 20 seconds of video-rate frames overlaid to show neutrophil tracks (green) in glomerular capillaries (red, Q-dot 565). Scale bars: $25 \mu \mathrm{m}$. (B) Distributions of cell dwell times in a single plane from video-rate recordings of control and $\alpha \mathrm{CBM}$ mice plotted in seconds. Duration times were significantly different between the groups using a 2-tailed Mann-Whitney $U$ test, $P<0.001$. (C) Distributions using a 2-sample KS test comparison percentile plot. Group dwell time distributions were significantly different, $P<0.001, D=0.6970$. (D and E) Neutrophil velocities were plotted over time and fit to a hidden Markov 2-state model (HMM 2-state) to identify cell track transitions from fast moving, state 1 (S1), to adherent, state 2 (S2), behavior. Two representative cell tracks (1 and 2) from the control group (D) with typical steady-state behavior showed fluctuations in cell velocity, but no S1 to S2 transition, while those from the $\alpha \mathrm{CBM}$ group (E) made brief S2 transitions (left panel) and sustained S2 transitions (right panel). (F) Mean dwell time \pm SEM in seconds from video-rate recordings in control and $\alpha$ GBM groups. (C) Average $S 1$ to $S 2$ transition frequency per track in control and $\alpha \mathrm{GBM}$ groups. $\mathrm{S} 2$ transitions were more frequent in the $\alpha \mathrm{GBM}$ group. (H) Average S2 durations were more prolonged in the $\alpha \mathrm{CBM}$ compared with the control group. (I) Average neutrophil S1 velocities ( $\mu \mathrm{m} / \mathrm{s}$ ) were higher in the control versus the $\alpha$ GBM group. ${ }^{*} P<0.01,{ }^{* *} P<0.001$, by 2-tailed unpaired $t$ test for F-I.

ular slice per hour at steady state and after anti-GBM antibody injection, respectively. The fraction of crawling neutrophils out of all trafficking cells was less than $0.2 \%$ (Supplemental Figure 4, D and E, and Supplemental Video 3).

In order to better characterize the dynamics of the short-duration neutrophil-vessel wall interactions in glomeruli, we imaged in a single optical plane at high temporal resolution ( $40 \mathrm{~ms}$ per frame) (Figure 6, A-I). Individual cell tracks in glomeruli of control mice were brief and had few periods of stable adhesion within glomeru- lar capillaries (Figure 6A, left panel, and Supplemental Video 4). In contrast, tracks in the anti-GBM-treated group were longer in duration and often had multiple periods of stable adhesion (Figure 6A, right panel, and Supplemental Video 5). We compared the distribution of video-rate duration times in control and anti-GBM groups in a single optical plane (Figure 6B). Similar to the 3D time-lapse data (Figure 5D), duration time distributions were heavily skewed toward shorter-duration interactions, but these events were better resolved. Control and anti-GBM groups were significantly dif- 
A

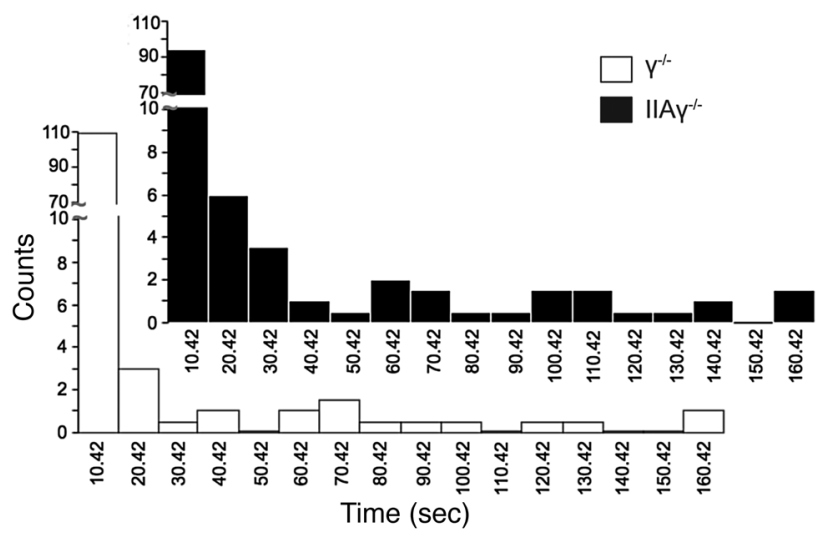

D

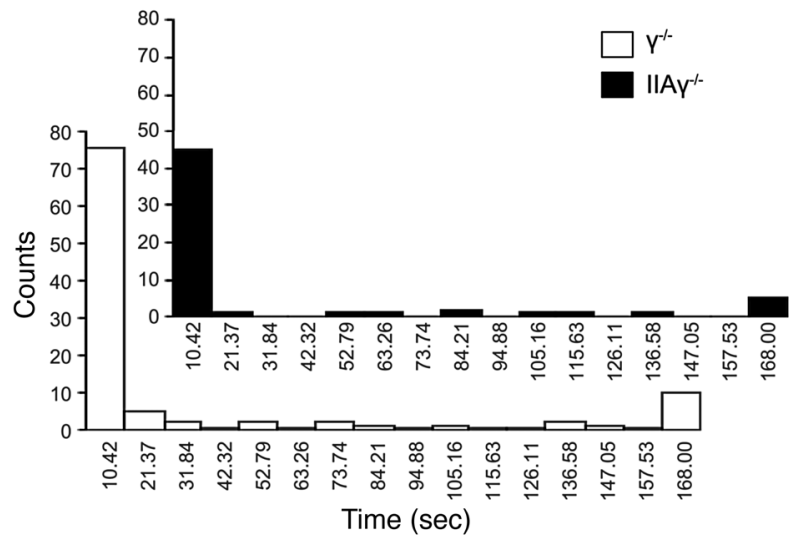

G

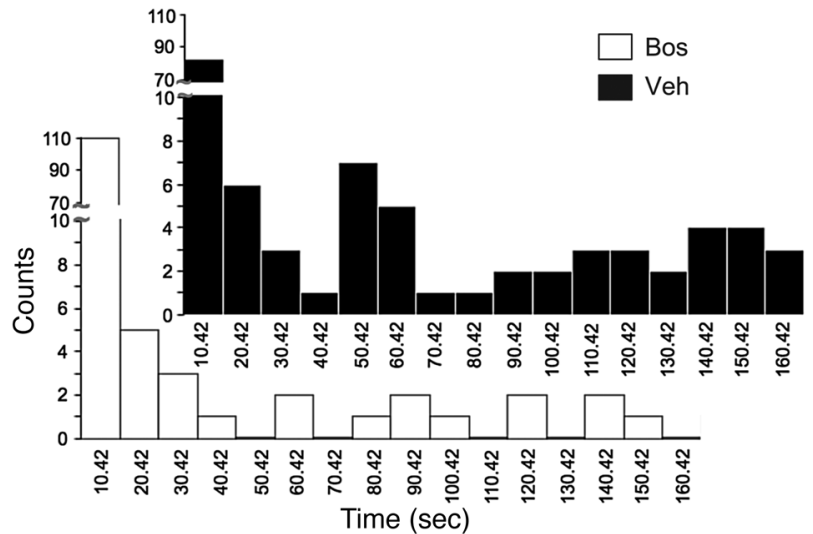

B

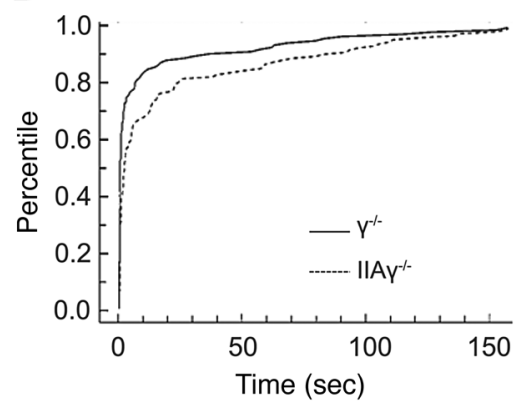

E

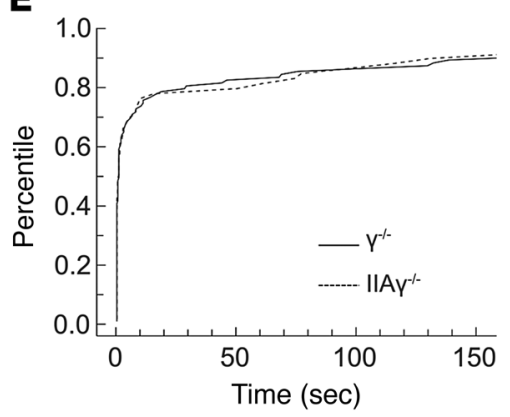

H

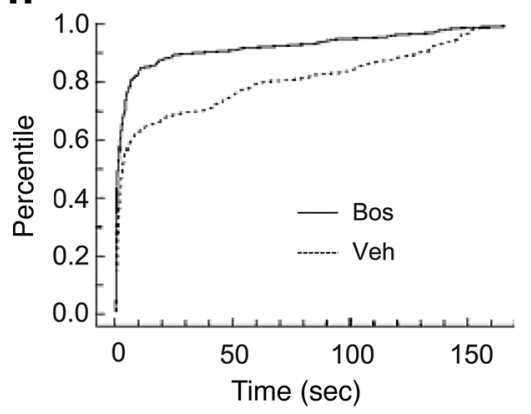

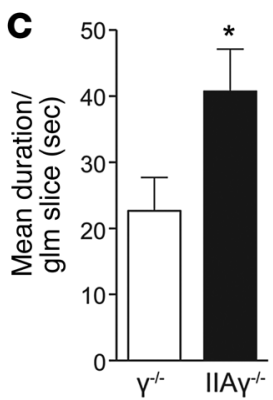

$\mathbf{F}$

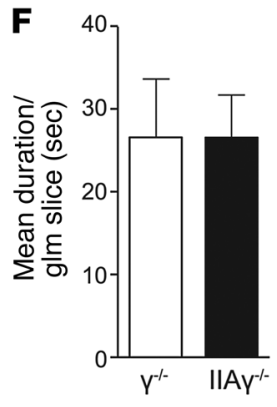

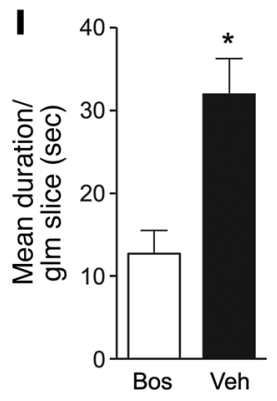

Figure 7. Rapid neutrophil capture in glomerular capillaries is FcyRIIA and AbI/Src dependent. (A-F) Fc $\gamma \mathrm{RIIA} / \gamma^{-/-}$recipient mice were given anti-CBM antibody $(\alpha \mathrm{CBM})(\mathbf{A}-\mathbf{C})$ or left untreated (D-F), and 2-photon time-lapse imaging of the kidney was conducted 2 hours later following the simultaneous adoptive transfer of Fc $\gamma \mathrm{RIIA}^{+} / \gamma^{-1-}\left(\mathrm{IIA} \gamma^{-1-}\right)$ and $\gamma^{-1-}$ neutrophils differentially labeled with green or blue fluorescent dye and a red dextran vascular dye. Duration times were calculated as the time in seconds that neutrophils remained arrested in the capillary and the distribution of dwell times plotted as histograms. (A-C) In $\alpha \mathrm{GBM}$-treated recipients given IIA $\gamma^{-1-}$ and $\gamma^{-1-}$ neutrophils, the group and dwell time distributions were compared using a Mann-Whitney $U$ test $(\mathbf{A}, P<0.001)$, a 2-sample KS test comparison percentile plot $(\mathbf{B}, P<0.001, D=0.2485)$, and a 2-tailed unpaired $t$ test $\left(\mathbf{C},{ }^{*} P<0.001\right)(n=4$ recipient mice with $n=2$ for each neutrophil dye combination, 33 glomeruli). (D-F) Untreated recipients were analyzed following the adoptive transfer of labeled $\| \mathrm{A} \gamma^{-1-}$ and $\gamma^{-1-}$ neutrophils, and statistics were conducted as in $\mathbf{A}-\mathbf{C}(\mathbf{D}, P=0.138 ; \mathbf{E}, P=0.138, D=0.1859 ; \mathbf{F}, P=0.138)(n=3$ recipient mice with 1 dye combination, 21 glomeruli). (G-I) Fc $\gamma \mathrm{RIIA} / \gamma^{-1-}$ recipient mice were given $\alpha \mathrm{CBM}$, and time-lapse imaging was conducted 2 hours after the adoptive transfer of differentially labeled $\mathrm{F} c \gamma \mathrm{RIIA} / \gamma^{-/-}$neutrophils pretreated with $100 \mathrm{nM}$ bosutinib (Bos) or vehicle (DMSO). Duration time (seconds) and group dwell time distributions were compared as in A-C. Statistics are as follows: $\mathbf{G}, P<0.001 ; \mathbf{H}, P<0.001$ and $D=0.2346 ; \mathbf{I},{ }^{*} P<0.001$ ( $n=4$ recipient mice with $n=2$ for each neutrophil dye combination, 22 glomeruli). Bar graphs in $\mathbf{C}, \mathbf{F}$, and I are mean duration time \pm SEM in seconds. 
A

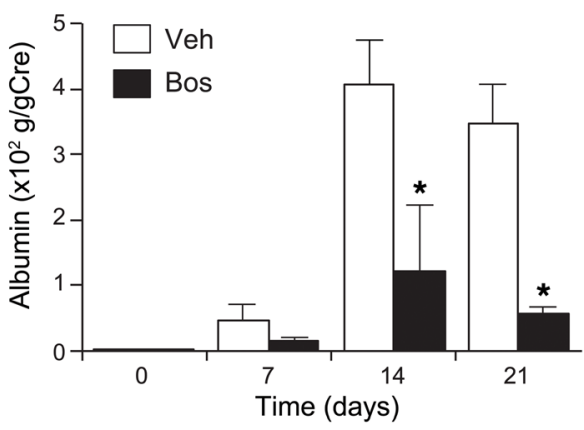

C

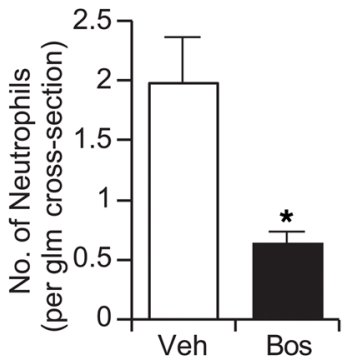

B

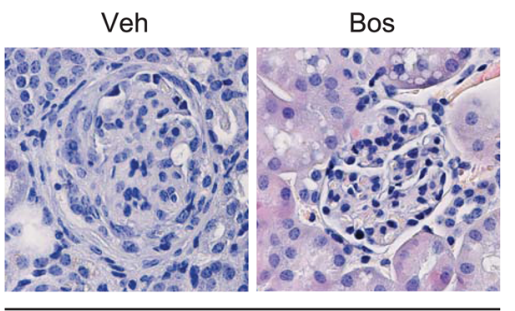

H\&E
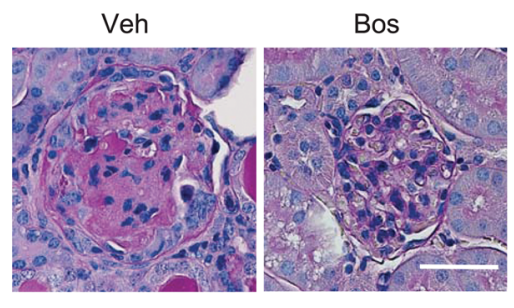

PAS
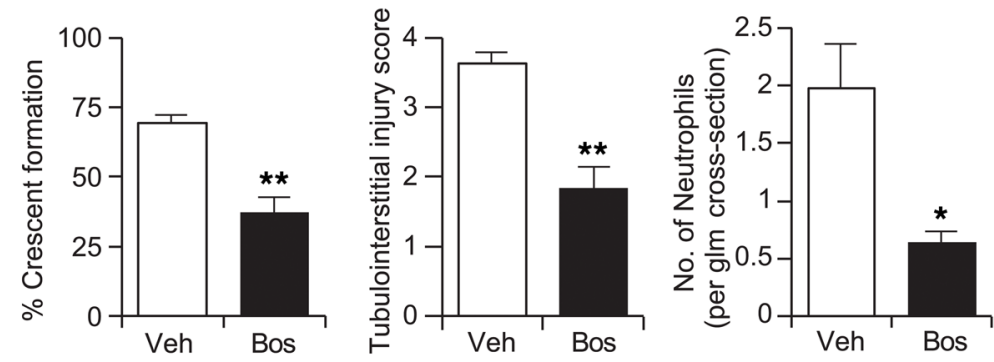

Figure 8. Bosutinib attenuates renal neutrophil accumulation and injury in crescentic anti-GBM nephritis. Fc $\gamma \mathrm{RIIA} / \gamma^{-/-}$mice preimmunized at day -3 were injected i.v. with anti-GBM ( $\alpha$ GBM) serum at day 0. Bosutinib (Bos, $150 \mathrm{mg} / \mathrm{kg}$ ) or vehicle (Veh) was administered by gavage every 24 hours from day 0 to day 21. (A) Urine albumin normalized to creatinine was determined. (B) The kidneys were harvested at day 21. Representative images of renal sections and histological scores are shown. PAS, periodic acid-Schiff. Scale bar: $100 \mu \mathrm{m} . n=8-15$ mice per group in A and B. (C) Clomerular (glm) neutrophil accumulation in anti-neutrophil antibody-stained renal sections was quantitated. $n=3$ for vehicle and $n=4$ mice for bosutinib. ${ }^{*} P<0.05$, ${ }^{*} P<0.01$, by 2 -tailed unpaired $t$ test.

ferent (2-tailed Mann-Whitney $U, P<0.01$ ), with the anti-GBM group having more frequent stable events lasting several seconds (Figure 6B). The duration time distributions were also compared using a 2-sample KS test, and the percentile plot showed that the distributions diverged early and the maximum difference was in the subsecond range $(P<0.001, D=0.6970)$ (Figure 6C). To better describe neutrophil retention behavior, we used a hidden Markov 2-state model (HMM 2-state) to evaluate neutrophil transitions from state 1 (S1; irregular flowing behavior) to state 2 (S2; transient adhesion behavior). In control mice, cell velocity varied over time, but transitions to S2 were rare and cells typically passed through the field of imaging displaying S1 behavior (Figure 6D). In contrast, neutrophil tracks in the anti-GBM-treated group often contained abrupt transitions from S1 to S2, indicating that cells were actively binding to the vessel wall (Figure 6E, left panels). Transient binding events were frequent, but in some cases, adhesive interactions lasted for the duration of the recording (Figure 6E, right panels). Tracks in the anti-GBM group had significantly increased mean duration times (Figure 6F). Furthermore, adhesive interactions (S2 transitions) were more frequent in the anti-GBM group, occurring approximately once on average per track (Figure 6G), and S2 adhesive events were longer in duration in comparison with the control group (Figure 6H). S1 velocities were higher in the control group than in the anti-GBM group (Figure 6I), possibly as a result of more frequent upstream S2 events in the latter. Neither group displayed sustained classic selectin-mediated rolling behavior reported in larger vessels, which is substantially slower and may not occur in narrow glomerular capillaries as it requires changes in shear stress in the midline versus the vessel wall. Notably, the short duration of our video-rate imaging experiments was designed to catch the earliest steps of neutrophil recruitment. The early, subsecond adhesive events that we describe herein may precede the previously reported glomerular intraluminal crawling behavior (ref. 40 and Supplemental Figure 4).

Rapid neutrophil capture within glomerular capillaries is dependent on FcrRIIA and inhibited by bosutinib. Our single-cell analysis shows that when anti-GBM antibody is deposited, the frequency of $\mathrm{S} 2$ stable interactions increases, as does the average duration of S2 adhesion events (from $<0.25$ in the steady state to $\sim 1.6$ seconds in the anti-GBM treatment group). Our video-rate analysis using KS percentile plots suggests that IgG-mediated capture events primarily occur on this short-duration time scale (subsecond to second), while longer cell retention times on the order of minutes are likely to involve other cell adhesion mechanisms. Based on this duration window, we evaluated the role of Fc $\gamma$ RIIA in neutrophil binding using a time resolution of 0.42 seconds, which should be sufficient to document the majority of Fc-mediated capture events. Moreover, we evaluated a potential cell-autonomous role for human Fc $\gamma$ RIIA in the capture of neutrophils using adoptive transfer approaches: Fc $\gamma \mathrm{RIIA}^{+} / \gamma^{-/}$recipient mice were given an i.v. injection of rabbit anti-GBM antibody, and 1.5 hours later injected i.v. with differentially labeled, fluorescent Fc $\gamma \mathrm{RIIA}{ }^{+} / \gamma^{-/}$and $\gamma^{-/-}$ bone marrow-derived mature neutrophils, which allowed for the analysis of 2 defined cell populations in the same glomerulus. The kidney was then surgically exteriorized, glomeruli were imaged, and neutrophil duration times in a single optical plane were evaluated. We observed a statistically significant difference in the distribution of neutrophil duration times between $\mathrm{Fc} \gamma \mathrm{RIIA}^{+} / \gamma^{-/}$and $\gamma^{-/-}$neutrophils (Mann-Whitney $U, P<0.001$ ) (Figure 7A). Neutrophil duration time was also analyzed using a KS test comparison 
percentile plot (Figure 7B), which showed group duration time distributions that were significantly different $(P<0.001, D=0.2485)$ with $\mathrm{Fc} \gamma \mathrm{RIIA}^{+} / \gamma^{-1-}$ neutrophils having increased mean duration times compared with $\gamma^{-/-}$(Figure 7C). The observed binding defect in injected $\gamma^{-/-}$neutrophils despite the presence of Fc $\gamma \mathrm{RIIA}^{+} / \gamma^{-/-}$ neutrophils demonstrates cell-autonomous roles for Fc $\gamma$ RIIA in neutrophil recruitment. Notably, there was no difference in the distribution of duration times between Fc $\gamma \mathrm{RIIA}^{+} / \gamma^{-/-}$and $\gamma^{-/-}$neutrophils injected into untreated recipient mice (Figure 7, D-F).

To evaluate the role of $\mathrm{Abl} / \mathrm{Src}$ in Fc $\gamma$ RIIA-dependent recruitment, Fc $\gamma \mathrm{RIIA}^{+} / \gamma^{-/-}$neutrophils were pretreated with either bosutinib or vehicle control ex vivo, differentially labeled, and adoptively transferred into $\mathrm{Fc} \gamma \mathrm{RIIA}^{+} / \gamma^{-/-}$recipient mice given anti-GBM antibody. Bosutinib treatment significantly altered neutrophil duration time distributions in glomerular capillaries compared with vehicle-treated counterparts (Mann-Whitney $U, P<0.001$; KS test, $P<0.001, \mathrm{D}=0.2346$ ) (Figure $7, \mathrm{G}$ and $\mathrm{H}$ ) and reduced the mean neutrophil duration time (Figure 7I and Supplemental Video 6). As observed in LysM-GFP mice, adoptively transferred neutrophils were rapidly captured in the absence of prior rolling or crawling (Supplemental Video 6).

Bosutinib inhibits neutrophil accumulation and glomerular injury in a model of proliferative GN. Neutrophil accumulation is associated with glomerular injury in rapidly progressive, proliferative crescentic anti-GBM nephritis in patients (5). This is modeled in mice by the administration of anti-GBM antibody and preimmunization with Freund's adjuvant and rabbit IgG, which leads to GN over a period of 14-21 days. Neutrophil influx and injury in this model is neutrophil FcrRIIA dependent (9). We first established an effective in vivo dose of bosutinib in mice. Four hours after gavage of $150 \mathrm{mg} / \mathrm{kg}$ bosutinib (41), peripheral blood neutrophils from mice exhibited a reduction in Fc $\gamma$ RIIA cross-linking-induced ROS generation ex vivo (Supplemental Figure 5A). This dose also prevented the acute, cutaneous reverse passive Arthus reaction (Supplemental Figure 5, B and C) induced by the i.v. injection of BSA and the s.c. injection of anti-BSA antibody. Next, Fc $\gamma$ RI$\mathrm{IA}^{+} / \gamma^{-1-}$ mice were subjected to crescentic, anti-GBM nephritis and given a daily gavage of bosutinib or vehicle over a 21-day period. Bosutinib markedly reduced albuminuria (Figure $8 \mathrm{~A}$ ), crescent formation, tubulointerstitial injury (Figure 8B), and glomerular neutrophil accumulation (Figure 8C) despite normal deposition of anti-GBM antibody (Supplemental Figure 5D) and peripheral blood leukocyte counts (Supplemental Figure 5E). Although systemic administration of bosutinib may affect several cell types, our previous finding that Fc $\gamma$ RIIA expression on neutrophils can restore glomerular neutrophil influx and injury in $\gamma^{-/-}$mice subjected to crescentic anti-GBM nephritis (9) suggests that bosutinib reduced disease primarily by targeting Fc $\gamma$ RIIA on neutrophils in Fc $\gamma$ RIIA $/ \gamma^{-/-}$animals.

\section{Discussion}

Our studies show that unlike the hierarchical cascade of neutrophil recruitment described in postcapillary venules that begins with rolling and subsequent adhesion of neutrophils to the activated endothelium, IgG-IC deposition within glomerular capillaries directly recruits neutrophils via their Fc $\gamma$ RIIA. Moreover, the observation that glomerular capillaries are similar in size to neutrophils suggests that neutrophils may circumferentially interact with $\operatorname{IgG}$ deposits as they pass though. This unconventional IgG-mediated neutrophil capture occurs via Fc $\gamma$ RIIA and is an active process, as bosutinib, an inhibitor of Abl/Src kinases, markedly inhibited the number and duration of capture events both in vitro and in vivo. Mechanistically, these capture events may be facilitated by Abl1/Src kinase regulation of F-actin polymerization, which strengthens Fc $\gamma$ R-IgG interactions when the cells experience mechanical force, as may be the case when neutrophils transit through glomerular capillaries. Intravital microscopy analysis suggests that captured neutrophils may be released into circulation after transient interaction or sustain adhesion. The latter could trigger the intravascular generation of ROS, proteases, and NETs, which could promote glomerular damage (40, 42, 43). Bosutinib's inhibition of IC-mediated cytotoxic functions in vitro suggests that it may both reduce neutrophil recruitment and dampen neutrophil cytotoxicity in vivo. Accordingly, in an experimental model of proliferative, crescentic anti-GBM nephritis, bosutinib significantly averted Fc $\gamma$ RIIA-mediated neutrophil accumulation and glomerular injury.

Intravital microscopy revealed that neutrophils make brief, subsecond interactions with the endothelium in the steady state, presumably due to irregular hydrodynamic flow through the small-diameter and tortuous glomerular capillary bed. However, following anti-GBM deposition, the frequency of stable interactions increased, as did the average duration of adhesion events. Fc $\gamma$ RIIA on neutrophils played a significant, cell-autonomous role in the rapid capture of neutrophils following anti-GBM antibody deposition, suggesting that Fc $\gamma$ RIIA participates in one of the earliest steps of autoimmune-mediated neutrophil accumulation in the kidney. Binding of cells under shear requires the rapid formation of longer-lived adhesive bonds called catch bonds that resist shear-induced detachment forces of flowing blood; catch bonds have been shown to occur with selectin/ligand pairs, which are known to promote neutrophil capture and rolling $(44,45)$. We demonstrate that Fc $\gamma$ RIIA-IgG forms catch bonds in vitro that may be reflected in vivo in the subsecond transition of flowing neutrophils to arrest in the presence of anti-GBM antibody. The observed frequent Fc $\gamma$ RIIA-mediated neutrophil interactions with anti-GBM antibody within capillaries may lead to CD18 integrin activation and thus substitute for selectin-mediated rolling, which facilitates integrin-mediated firm adhesion (46). Indeed, activated Mac-1 sustains Fc $\gamma$ R-mediated neutrophil adhesion under static and flow conditions $(10,21,22)$ and following acute anti-GBM nephritis $(21,40)$. Interestingly, Mac-1 activation by ICs but not GPCR agonists (e.g., FMLP) relies on actin polymerization (47), which suggests the possibility that F-actin polymerization, essential for Fc $\gamma$ R-mediated neutrophil capture and for catch bond formation in vitro, may concurrently promote Mac-1 activation.

Although the molecular mechanisms driving Fc $\gamma$ R-mediated neutrophil cytotoxic functions such as phagocytosis and ROS generation are relatively well defined $(48,49)$, those that support Fc $\gamma$ R-mediated neutrophil capture by ICs were unknown. Our studies revealed a role for $\mathrm{Abl} / \mathrm{Src}$ in rapid neutrophil capture by ICs under flow conditions. The significant effect of Abl1 silencing on FcrRIIA-mediated neutrophil capture and F-actin polymerization contrasts with the partial effects of silencing Abl family mem- 
bers on other myeloid functions $(50,51)$ such as $\mathrm{Fc} \gamma \mathrm{R}$ phagocytosis (50), and emphasizes the bias in signaling pathways in different myeloid functions. Bosutinib also inhibited Fc $\gamma$ R-mediated cytotoxic functions, which is consistent with a study using a related $\mathrm{Abl} / \mathrm{Src}$ inhibitor, dasatinib, though in this case IC-mediated ROS generation and granule release were evaluated only under adhesion-dependent conditions (52), which are both Fc $\gamma \mathrm{R}$ and Mac-1 dependent (21). We specifically placed Abl1 kinases downstream of Fc $\gamma$ RIIA by examining bosutinib's effects after cross-linking Fc $\gamma$ RIIA in suspension, by treating neutrophils with other Abl inhibitors that do not affect Src kinases, and by Abl1 silencing. Abl1 silencing did not affect bolished Fc $\gamma$ RIIA-induced F-actin polymerization. Src inhibition also affected F-actin polymerization, suggesting the possibility that $\mathrm{Abl}$ and $\mathrm{Src}$ kinases are in the same pathway. Indeed, Src kinases may tyrosine-phosphorylate Abl1 to stabilize Abl's active conformation (54). On the other hand, the 2 kinases could act in parallel, as Src may contribute to the activation of PI3K, FAK-Pyk-2 kinases, and Vav that feed into the Rho GTPase-WASP pathway of actin polymerization (53), while Abl1 may directly bind F-actin and/or phosphorylate cytoskeletal proteins that regulate actin polymerization (19). Surprisingly, while actin polymerization was essential for Fc $\gamma$ RIIA-mediated cell capture on ICs under shear flow, it was largely dispensable for cell attachment to ICs under static conditions. Shear changes the biophysics of adhesion, as it requires the rapid formation of longer-lived adhesive bonds called catch bonds that resist shear-induced detachment forces (45). Indeed, catch bonds may increase neutrophil "on" rates while decreasing "off" rates and thereby increase neutrophil capture in the glomerulus following anti-GBM deposition.

The efficacy of bosutinib in reducing IgG-mediated glomerular neutrophil recruitment and associated injury suggests the possibility of repositioning this drug for the treatment of IgG-mediated proliferative $\mathrm{GN}$, which has significant unmet clinical need $(1,55,56)$. Glomerular IC deposition and neutrophil accumulation contribute to the pathogenesis of Goodpasture's syndrome (antiGBM disease) and acute poststreptococcal nephritis, which is a risk factor for subsequent development of chronic renal failure (1, 2). Neutrophils also accumulate in proliferative lupus nephritis (3), with recent transcriptional profiling of blood from lupus patients showing a gradual enrichment of neutrophil transcripts during progression to active nephritis (57). Reducing the renal burden and cytotoxic function of neutrophils may also preserve long-term kidney function by dampening intrarenal autoimmunity in predisposed individuals $(3,58,59)$. As with other compounds in the Bcr-Abl class of inhibitors, bosutinib can also target other kinases such as salt-inducible kinases and TEC-family kinases (32) and very likely has antiinflammatory effects beyond the inhibition of Fc $\gamma$ RIIA and neutrophils $(60,61)$. On the other hand, Src inhibition could promote dendritic cell activation as shown in vitro with dasatinib (62) or lead to lupus-like symptoms as reported in mice with B cell deficiency in the Src kinase Lyn (63). Indeed, the choice of Abl inhibitors may be important in limiting certain unwanted effects. For example, unlike dasatinib, bosutinib does not mobilize cytotoxic lymphocytes (64). Notably, bosutinib is a relatively well-tolerated drug (16). The serious adverse events are primarily early-onset gastrointestinal toxicity, mainly diarrhea, nausea, and vomiting that were low-grade, transient, and manageable (16).
In summary, our studies have revealed a new pathway of neutrophil recruitment in the glomerulus that depends on Fc $\gamma$ RIIA-mediated neutrophil capture by IgG deposited within glomerular capillaries. This may occur through Fc $\gamma$ RIIA-mediated Abl1 and Src kinase-induced F-actin polymerization, which in turn may strengthen Fc $\gamma$ RIIA-IgG interactions under flow conditions. We also provide evidence that bosutinib, currently used for the treatment of CML (65), averts this very early step in glomerular inflammation and may thus potentially be repurposed for Goodpasture's syndrome and possibly other proliferative GN.

\section{Methods}

Mice. Human Fc $\gamma$ RIIA-expressing, $\gamma$ chain-deficient $\left(\right.$ Fc $\left._{\text {RIIA }}{ }^{+} / \gamma^{-1}\right)$ mice and Fc $\gamma$ RIIA-expressing, $\gamma$ chain-deficient mice were as previously described $(9,66)$. These mice were maintained in a virus- and antibody-free facility at the New Research Building animal housing facility at Harvard Medical School. Mice used for each experiment were $8-10$ weeks of age and were age- and sex-matched. LysM-GFP mice were provided by Klaus Ley (La Jolla Institute for Allergy and Immunology, La Jolla, California, USA), who backcrossed the originally generated mice from T. Graf (Albert Einstein College of Medicine, Bronx, New York, USA) to the B6 background. These mice were maintained and bred under specific pathogen-free conditions at Washington University School of Medicine. The Harvard Medical Association IACUC at Harvard Medical School approved all animal experiments.

Neutrophil ROS-based high-throughput small-molecule screen. In collaboration with the Institute of Chemistry and Cell Biology Screening facility at Harvard Medical School (ICCB-Longwood), 8,483 compounds were screened. Details are in Supplemental Methods. For a secondary screen, serial dilutions of the 30 top-ranked compounds that suppressed ROS generation were tested on a single 384-well plate using the same protocol as the primary screen. Reagents for subsequent studies were as follows: cytochalasin D and PP2 from Sigma-Aldrich and bosutinib (B1788), imatinib (I5508), and nilotinib (N8207) from LC Laboratories.

Human neutrophils, mouse bone marrow neutrophils, and HL-60 cells. Human polymorphonuclear neutrophils (>95\% pure) were isolated from whole blood drawn from healthy volunteers as previously described (67) under Partners IRB approval (protocol 1999P001694). Mouse bone marrow neutrophils (BMNs) were isolated by Percoll gradient separation. HL-60 cells (ATCC) were cultured in RPMI 1640 supplemented with 10\% FBS, L-glutamine, and penicillin/streptomycin. The cells were differentiated into neutrophils with $0.8 \%$ dimethylformamide (DMF; Sigma-Aldrich) for 4 days. Sequence-verified shRNA lentiviral plasmids against Abl1 (Mission library, Sigma-Aldrich, TRCNO000121100 and TRCNO000121101) were used to produce lentiviral particles in HEK293 T cells (clone 17, ATCC) by standard methods. Undifferentiated HL-60 cells were transduced with lentiviral shRNA particles, cultured for 5 days, selected for 10 days with $10 \mu \mathrm{g} / \mathrm{ml}$ puromycin, and differentiated for an additional 4 days with DMF.

Human CML blood samples. Anticoagulated blood samples (collected in citrate buffer) were obtained from 3 patients on 200, 400, or 500 mg/d bosutinib and age-, sex-, and ethnicity-matched normal volunteers. The blood was subjected to 2-3 sequential rbc lysis steps using ammonium-chloride-potassium (ACK) lysis buffer, resuspended in PBS at $1 \times 10^{7}$ cells $/ \mathrm{ml}$, and adjusted to $1 \times 10^{6}$ cells with RPMI plus $0.1 \%$ 
BSA before experiments. Aliquots were evaluated by FACS analysis for Fc $\gamma$ RIIA expression using anti-CD32 FITC (555448, BD Biosciences). Granulocytes were identified by forward scatter and side scatter.

Fc $\gamma$ R cross-linking-induced generation of ROS, neutrophil degranulation, and Western blot analysis. Additional details and the complete unedited Western blots are provided in the supplemental materials.

Adhesion and spreading assay under static conditions. The adhesion assay and F-actin staining were as previously described (21). Briefly, neutrophils were plated on glass coverslips coated with BSA and antiBSA (B7276, Sigma-Aldrich), and coverslips were fixed 20 minutes later. Adherent cell numbers were quantitated in 3 independent fields at magnification $\times 200$, and the average cell number per field was determined. Cells were stained with Alexa Fluor 568 phalloidin, the area of a single spread cell was calculated with Image (NIH), and the values of 20 cells were averaged.

Adhesion assay under shear stress. For binding assay on ICs, coverslips were coated with BSA or preformed soluble ICs of BSA-anti-BSA for 2 hours at room temperature, which were generated as previously described $(10,68)$. Coverslips were mounted on a flow chamber, and cells were perfused for 4.5 minutes at the indicated shear stresses. Live imaging of cell adhesion was recorded with a video camera on a Nikon TE2000 inverted microscope equipped with a $20 \times 0.75$ NA phase contrast objective and VideoLab software (Mitov).

Human dermal microvascular endothelial cells (HDMECs) (HMVEC-D, Lonza) were grown to confluence on fibronectin-treated glass coverslips and activated with human TNF- $\alpha(10 \mathrm{ng} / \mathrm{ml})$ for 4 hours where indicated. ECs were incubated with mouse anti-endoglin $\mathrm{mAb}$ (anti-CD105, clone 43A3, BioLegend) for 15 minutes at $37^{\circ} \mathrm{C}$, gently washed, and subsequently incubated with rabbit anti-mouse IgG (1:250 dilution) for 15 minutes. HDMECs were assembled into a parallel plate flow chamber maintained at $37^{\circ} \mathrm{C}$. Human peripheral PMNs were purified from freshly drawn blood and incubated with anti-Fc $\gamma$ RIIIB (clone 3G8, BioLegend), anti-Fc $\gamma$ RIIA (clone IV.3, Stemcell Technologies), appropriate isotype (Iso) controls or bosutinib $(100 \mathrm{nM})$, imatinib $(100 \mathrm{nM})$, or cytochalasin $\mathrm{D}(20 \mu \mathrm{M})$ for $30 \mathrm{~min}$ utes at $37^{\circ} \mathrm{C}$. The human PMNs were diluted 10 -fold to $10^{6}$ cells $/ \mathrm{ml}$ and drawn across the endothelial cell layer at $1.5 \mathrm{dyn} / \mathrm{cm}^{2}$. For all flow experiments, the number of cells that accumulated in 4 fields in each coverslip was calculated and averaged. For each independent experiment, 2 coverslips were evaluated per condition and averaged.

Flow cytometric analysis of F-actin polymerization. $10 \times 10^{6} \mathrm{HL}-60$ cells $/ \mathrm{ml}$ in PBS were incubated with $10 \mu \mathrm{g} / \mathrm{ml}$ mouse anti-hFc $\gamma$ RIIA (clone IV.3, Stemcell Technologies) and thereafter stained with Zombie Aqua (423102, BioLegend) on ice for 20 minutes. After washing, cells were incubated with $1 \mathrm{ng} / \mathrm{ml}$ human GM-CSF (R\&D Systems) in PBS with $\mathrm{Ca}^{2+} / \mathrm{Mg}^{2+}$ at $37^{\circ} \mathrm{C}$ for 15 minutes and pretreated with indicated reagents at $37^{\circ} \mathrm{C}$ for 30 minutes. Goat anti-mouse $\mathrm{F}\left(\mathrm{ab}^{\prime}\right)_{2}(36 \mu \mathrm{g} / \mathrm{ml} ; 112$ 006-072, Jackson ImmunoResearch) was added to induce cross-linking, and the reaction was stopped with ice-cold $1.5 \%$ PFA solution at $4^{\circ} \mathrm{C}$ for 30 minutes. After permeabilization with $0.1 \%$ saponin (SigmaAldrich) for 2 minutes at room temperature, cells were stained with NBD-phallacidin (Life Technologies) at $4^{\circ} \mathrm{C}$ for 20 minutes, and the fluorescence signal intensity was analyzed with a DxP12 Analyzer (Cytek).

$r b c$ and $h I g G 1 /$ streptavidin bead preparation for biomembrane force probe experiments. Human rbc were isolated from whole blood and biotinylated as previously described (69), and hIgG1/streptavidin-coated beads were prepared as detailed in Supplemental Methods.
Biomembrane force probe experiments. The biomembrane force probe technique has been previously described (69). In a chamber filled with $\mathrm{L} 15$ buffer with $1 \%$ BSA and $1 \mathrm{mM} \mathrm{Ca}^{2+} / \mathrm{Mg}^{2+}$, a hIgG1/streptavidin bead was attached to the apex of a micropipette-aspirated biotinylated rbc, which together acted as an ultrasensitive force transducer. The aspiration pressure was set to control the probe stiffness at 0.2 or 0.3 $\mathrm{pN} / \mathrm{nm}$, based on calculations (70). The axial displacement of the beadrbc interface was monitored by high-speed camera, which reflected the force on the rbc by Hooke's law. Jurkat cells lacking CD18 integrins (36), provided by Timothy A. Springer (Harvard Medical School, Boston, Massachusetts, USA), were engineered to express Fc $\gamma$ RIIA. The Jurkat-Fc $\gamma$ RIIA-expressing cell was then aspirated by an opposing micropipette and driven by a piezo actuator with subnanometer spatial precision. The Jurkat cell was programmed to approach and briefly interact with the probe bead to allow bond formation, which was signified by a tensile force on the rbc upon retraction of the cell. Following that, distance clamping was performed to measure the lifetime of this bond under a certain force. After dissociation of the bond, the cell was retracted to the original position to wait for the next cycle. To measure the bond lifetimes under zero force, after touching the bead, the Jurkat-Fc $\gamma$ RIIAexpressing cell was retracted to the zero-force position and then held stationary. Bond association/dissociation was manifested as a sudden drop/increase in the thermal fluctuations of the bead position, which was quantified by the average standard deviation of a sliding interval of 70 time points (71). The site density of the hIgG1 was titrated to keep the adhesion frequency approximately $20 \%$, to ensure that approximately $90 \%$ of adhesion events were mediated by single bonds. As a confirmation of the binding specificity, beads coated with streptavidin alone contacting the same batch of Jurkat cells only rendered approximately $3 \%$ adhesion frequency. For experiments with cytochalasin D, Jurkat-expressing Fc $\gamma$ RIIA cells were incubated with $20 \mu \mathrm{M}$ of cytochalasin D for 30 minutes under $37^{\circ} \mathrm{C}$ before being placed into the chamber.

Two-photon intravital microscopy of the glomerulus. Fc $\gamma \mathrm{RIIA}^{+} / \gamma^{-1-}$ isolated mouse BMNs $\left(1 \times 10^{7}\right)$ were stained with Cell Tracker Green CMFDA or Cell Tracker Blue CMF2HC ( $5 \mu \mathrm{M}$; Life Technologies), washed with PBS, and incubated with $100 \mathrm{nM}$ bosutinib or DMSO vehicle, respectively, at $37^{\circ} \mathrm{C}$ for 30 minutes. A recipient Fc $\gamma R \mathrm{RIIA}^{+} / \gamma^{-1-}$ mouse was given anti-GBM serum, and labeled neutrophils were transferred i.v. Alternatively, LysM-GFP reporter mice were given anti-GBM serum to image endogenous neutrophil trafficking during glomerular injury and in some cases additionally given a retro-orbital injection of $3.5 \mu \mathrm{g}$ of PE-anti-Ly6G antibody (clone 1A8, eBioscience). The right kidney of the mouse was surgically exteriorized 1.5 hours later and prepared for intravital microscopy, which took 30 minutes. Details for intravital microscopy imaging are in Supplemental Methods. Glomerular lumen diameter was measured manually in z-sections using the Imaris 8.2 line measurement tool. The diameter of LysM-GFP neutrophils in glomerular capillaries was measured along the smaller axis. For all intravital microscopy experiments, 2 or more glomeruli were analyzed per mouse.

Two-photon imaging data analysis and the HMM 2-state model. Velocities were plotted over time and fit to an HMM 2-state plot. The rationale for using an HMM 2-state model stems from the fact that neutrophils do not flow smoothly through glomerular capillaries in the steady state. This unusual behavior may in part be due to irregularities in hydrodynamic flow in small tortuous vessels, interactions with other cells in the capillaries, or adhesive interactions independent of Fc $\gamma$ Rs. The HMM 2-state model allows us to discriminate fluctuations in velocity and tran- 
sitory adhesive interactions that are common in the steady-state neutrophil behavior (defined as $\mathrm{S} 1$ behaviors) from bona fide Fc $\gamma \mathrm{R}$-dependent binding events in anti-GBM-treated mice. We used the HMM 2-state to assign cell decelerations from $\mathrm{S} 1$ as coming from the "hidden state" (i.e., S2) when track speed drops below $\leq 50 \mu \mathrm{m} / \mathrm{s}$ for 2 consecutive frames, which filters out steady-state fluctuations and respiration artifacts. S2 was defined as a genuine binding interaction and presumably occurred as a result of Fc $\gamma R$ catch bond formation. Track durations were obtained and plotted to demonstrate the number of cells at time intervals of 0.56 seconds for each group. Average velocities per individual track from control and anti-GBM groups were calculated, along with the average duration spent in each state (S1, S2). In order to demonstrate the difference in neutrophil behavior between the control and anti-GBM, the average frequency of neutrophil adhesion in kidney glomeruli was plotted. The mean \pm SEM or median \pm median absolute deviation was calculated for distributions as descriptive statistics, and reported for non-normal distributions to provide a rough value for discussion. Group distributions were plotted as histograms, and non-normal data were compared using a nonparametric 2-tailed Mann-Whitney $U$ test where a confidence interval of $95 \%$ was used to determine statistical significance. In addition, a 2-sample Kolmogorov-Smirnov (KS) test was performed to compare non-normal distributions. The KS test identifies distances between distributions, and the maximum distance $(D)$ is shown to asymptotically converge to a statistical distribution of the exponential family and thus can assign a probability that the 2 distributions are equivalent. The maximum distance $D$ is reported for each test, and the $P$ value associated with the distance is also reported; a confidence interval of $95 \%$ was used to determine statistical significance.

Cutaneous reverse passive Arthus reaction. The reverse passive Arthus reaction was induced in the dorsal skin, and neutrophil accumulation and edema were evaluated as we have described (72). Details are provided in the Supplemental materials.

Nephritis model. Proliferative, crescentic anti-GBM glomerulonephritis was induced and neutrophil accumulation and renal injury were evaluated as we have described (73). Details are provided in Supplemental Methods.

Statistics. All in vitro data and in vivo disease models are presented as the mean \pm SEM. One-way ANOVA followed by Dunnett's test was used for multiple comparisons. Kidney histology scoring and infiltrated neutrophil counts were compared by the Mann-Whitney $U$ test.
For other data, statistical differences were analyzed with the unpaired 2-tailed $t$ test. $P$ less than 0.05 was considered significant. For statistical analysis of data presented as fold induction, an average of data from control samples is assigned a value of 1 , and all data are calculated relative to this value.

Study approval. The Harvard Medical School Animal Care and Use Committee approved all procedures in this study. Human peripheral blood was obtained from healthy volunteers under a protocol approved by the Brigham and Women's Hospital IRB, and all subjects gave written informed consent. Blood samples from CML patients were obtained under a Dana-Farber Cancer Institute IRB-approved protocol.

\section{Author contributions}

$\mathrm{HN}, \mathrm{XC}, \mathrm{KF}, \mathrm{GS}, \mathrm{YC}, \mathrm{FR}$, JL, and JMH conducted the experiments and acquired and analyzed the data. SLH, LY, SPP, and MJM conducted 2-imaging experiments, analyzed data, and edited the manuscript, and JCB performed calculations and provided guidance on the statistical analysis of the intravital microscopy data. DJD provided blood samples from CML patients. TNM, HN, and $\mathrm{XC}$ wrote the manuscript. TNM, HN, XC, KF, GCT, and CZ designed the research studies.

\section{Acknowledgments}

We thank the Harvard Medical School Center for Immune Imaging Core (director: Ulrich von Andrian) for advice and use of their 2-photon intravital microscope. This work was supported by a Target Identification in Lupus Grant/Alliance for Lupus Research Foundation grant (to TNM) and a Distinguished Innovator Award/ Lupus Research Initiative (to TNM); NIH HL065095 (to TNM), DK099507 (to TNM), AI42269 (to GCT), AI044902 (to CZ), DK097317 (to MJM), AI095776 (to MJM), AI077600 (to MJM), and T32 HL007627 (to FR); and a Japan Society for the Promotion of Science Postdoctoral Fellowship for Research Abroad (to HN).

Address correspondence to: Tanya N. Mayadas, Department of Pathology, Brigham and Women's Hospital and Harvard Medical School, 77 Avenue Louis Pasteur, NRB Room 752O, Boston, Massachusetts 02115, USA. Phone: 617.525.4336; Email: tmayadas@ rics.bwh.harvard.edu.
1. Chan AL, Louie S, Leslie KO, Juarez MM, Albertson TE. Cutting edge issues in Goodpasture's disease. Clin Rev Allergy Immunol. 2011;41(2):151-162.

2. Rodriguez-Iturbe B, Haas M. Post-streptococcal glomerulonephritis. In: Ferretti JJ, Stevens DL, Fischetti VA eds. Streptococcus pyogenes: Basic Biology to Clinical Manifestations. Oklahoma City, Oklahoma, USA; University of Oklahoma Health Sciences Center: 2016.

3. Smith CK, Kaplan MJ. The role of neutrophils in the pathogenesis of systemic lupus erythematosus. Curr Opin Rheumatol. 2015;27(5):448-453.

4. Jennette JC, Falk RJ. Pathogenesis of antineutrophil cytoplasmic autoantibody-mediated disease. Nat Rev Rheumatol. 2014;10(8):463-473.

5. Mayadas TN, Rosetti F, Ernandez T, Sethi S. Neutrophils: game changers in glomerulonephritis? Trends Mol Med. 2010;16(8):368-378.

6. Nimmerjahn F, Ravetch JV. Fc $\gamma$ receptors as reg- ulators of immune responses. Nat Rev Immunol. 2008;8(1):34-47.

7. Tarzi RM, Cook HT. Role of Fc $\gamma$ receptors in glomerulonephritis. Nephron Exp Nephrol. 2003;95(1):e7-12.

8. Bruhns P, Jönsson F. Mouse and human FcR effector functions. Immunol Rev. 2015;268(1):25-51.

9. Tsuboi N, Asano K, Lauterbach M, Mayadas TN. Human neutrophil Fcy receptors initiate and play specialized nonredundant roles in antibody-mediated inflammatory diseases. Immunity. 2008;28(6):833-846.

10. Coxon A, et al. Fc $\gamma$ RIII mediates neutrophil recruitment to immune complexes. a mechanism for neutrophil accumulation in immune-mediated inflammation. Immunity. 2001;14(6):693-704.

11. Florey OJ, Johns M, Esho OO, Mason JC, Haskard DO. Antiendothelial cell antibodies mediate enhanced leukocyte adhesion to cytokine-activated endothelial cells through a novel mechanism requiring cooperation between Fc $\gamma$ RIIa and CXCR1/2. Blood. 2007;109(9):3881-3889.

12. Stan RV, Kubitza M, Palade GE. PV-1 is a component of the fenestral and stomatal diaphragms in fenestrated endothelia. Proc Natl Acad Sci U S A. 1999;96(23):13203-13207.

13. Meroni PL, D'Cruz D, Khamashta M, Youinou P, Hughes GR. Anti-endothelial cell antibodies: only for scientists or for clinicians too? Clin Exp Immunol. 1996;104(2):199-202.

14. Hudson BG, Tryggvason K, Sundaramoorthy M, Neilson EG. Alport's syndrome, Goodpasture's syndrome, and type IV collagen. $N$ Engl J Med. 2003;348(25):2543-2556.

15. Cortes J. Bosutinib in the treatment of chronic myelogenous leukemia. Clin Adv Hematol Oncol. 2012;10(11):736-737. 
16. Kantarjian HM, et al. Bosutinib safety and management of toxicity in leukemia patients with resistance or intolerance to imatinib and other tyrosine kinase inhibitors. Blood 2014;123(9):1309-1318.

17. Lowell CA. Src-family and Syk kinases in activating and inhibitory pathways in innate immune cells: signaling cross talk. Cold Spring Harb Perspect Biol. 2011;3(3):a002352.

18. Futosi K, Fodor S, Mócsai A. Reprint of Neutrophil cell surface receptors and their intracellular signal transduction pathways. Int Immunopharmacol. 2013;17(4):1185-1197.

19. Wang JY. The capable ABL: what is its biological function? Mol Cell Biol. 2014;34(7):1188-1197.

20. Nathan C, et al. Cytokine-induced respiratory burst of human neutrophils: dependence on extracellular matrix proteins and CD11/CD18 integrins. JCell Biol. 1989;109(3):1341-1349.

21. Tang T, et al. A role for Mac-1 (CDIIb/CD18) in immune complex-stimulated neutrophil function in vivo: Mac-1 deficiency abrogates sustained Fcgamma receptor-dependent neutrophil adhesion and complement-dependent proteinuria in acute glomerulonephritis. J Exp Med. 1997;186(11):1853-1863.

22. Jones SL, Brown EJ. Functional cooperation between Fcgreceptors andcomplement receptors in phagocytes. In: van de Winkel JGJ, Capel PJA, eds. Human IgG Fc Receptors. Austin, Texas, USA: RG Landes Co.; 1996:149-163.

23. Yalamanchili P, Lu C, Oxvig C, Springer TA. Folding and function of I domain-deleted Mac- 1 and lymphocyte function-associated antigen-1.J Biol Chem. 2000;275(29):21877-21882.

24. Nauseef WM, Volpp BD, McCormick S, Leidal $\mathrm{KG}$, Clark RA. Assembly of the neutrophil respiratory burst oxidase. Protein kinase $\mathrm{C}$ promotes cytoskeletal and membrane association of cytosolic oxidase components. J Biol Chem. 1991;266(9):5911-5917.

25. Chen K, et al. Endocytosis of soluble immune complexes leads to their clearance by Fc $\gamma$ RIIIB but induces neutrophil extracellular traps via Fc $\gamma$ RIIA in vivo. Blood. 2012;120(22):4421-4431.

26. Behnen M, et al. Immobilized immune complexes induce neutrophil extracellular trap release by human neutrophil granulocytes via Fc $\gamma$ RIIIB and Mac-1. J Immunol. 2014;193(4):1954-1965.

27. Alemán OR, Mora N, Cortes-Vieyra R, UribeQuerol E, Rosales C. Differential use of human neutrophil Fcy receptors for inducing neutrophil extracellular trap formation. J Immunol Res. 2016;2016:2908034.

28. Quintás-Cardama A, Kantarjian H, Cortes J. Flying under the radar: the new wave of BCR-ABL inhibitors. Nat Rev Drug Discov. 2007;6(10):834-848.

29. Zhou MJ, Lublin DM, Link DC, Brown EJ. Distinct tyrosine kinase activation and Triton X-100 insolubility upon Fc gamma RII or Fc gamma RIIIB ligation in human polymorphonuclear leukocytes. Implications for immune complex activation of the respiratory burst. J Biol Chem. 1995;270(22):13553-13560.

30. Ley K, Laudanna C, Cybulsky MI, Nourshargh S. Getting to the site of inflammation: the leukocyte adhesion cascade updated. Nat Rev Immunol. 2007;7(9):678-689.
31. de Jong R, ten Hoeve J, Heisterkamp N, Groffen $\mathrm{J}$. Tyrosine 207 in CRKL is the BCR/ABL phosphorylation site. Oncogene. 1997;14(5):507-513.

32. Remsing Rix LL, et al. Global target profile of the kinase inhibitor bosutinib in primary chronic myeloid leukemia cells. Leukemia. 2009;23(3):477-485.

33. Woodring PJ, Hunter T, Wang JY. Regulation of F-actin-dependent processes by the Abl family of tyrosine kinases. J Cell Sci. 2003; 116(pt 13):2613-2626.

34. Brennan PJ, Zigmond SH, Schreiber AD, Smith ER, Southwick FS. Binding of IgG containing immune complexes to human neutrophil Fc $\gamma$ RII and Fc $\gamma$ RIII induces actin polymerization by a pertussis toxin-insensitive transduction pathway. Jimmunol. 1991;146(12):4282-4288.

35. Zhu C, McEver RP. Catch bonds: physical models and biological functions. Mol Cell Biomech. 2005;2(3):91-104.

36. Weber KS, York MR, Springer TA, Klickstein LB. Characterization of lymphocyte function-associated antigen 1 (LFA-1)-deficient $T$ cell lines: the $\alpha \mathrm{L}$ and $\beta 2$ subunits are interdependent for cell surface expression. JImmunol. 1997;158(1):273-279.

37. Kreisel D, et al. In vivo two-photon imaging reveals monocyte-dependent neutrophil extravasation during pulmonary inflammation. Proc Natl Acad Sci U S A. 2010;107(42):18073-18078.

38. Wang $\mathrm{B}$, et al. In vivo imaging implicates CCR2(+) monocytes as regulators of neutrophil recruitment during arthritis. Cell Immunol. 2012;278(1-2):103-112.

39. Zinselmeyer BH, et al. Chapter 16. Two-photon microscopy and multidimensional analysis of cell dynamics. Meth Enzymol. 2009;461:349-378.

40. Devi S, et al. Multiphoton imaging reveals a new leukocyte recruitment paradigm in the glomerulus. Nat Med. 2013;19(1):107-112.

41. Golas JM, et al. SKI-606, a Src/Abl inhibitor with in vivo activity in colon tumor xenograft models. Cancer Res. 2005;65(12):5358-5364.

42. Schrijver G, Schalkwijk J, Robben JC, Assmann KJ, Koene RA. Antiglomerular basement membrane nephritis in beige mice. Deficiency of leukocytic neutral proteinases prevents the induction of albuminuria in the heterologous phase. JExp Med.1989;169(4):1435-1448.

43. Kumar SV, et al. Neutrophil extracellular trap-related extracellular histones cause vascular necrosis in severe GN. J Am Soc Nephrol. 2015;26(10):2399-2413.

44. Yusko EC, Asbury CL. Force is a signal that cells cannot ignore. Mol Biol Cell. 2014;25(23):3717-3725

45. McEver RP, Zhu C. Rolling cell adhesion. Annu Rev Cell Dev Biol. 2010;26:363-396.

46. Zarbock A, Ley K, McEver RP, Hidalgo A. Leukocyte ligands for endothelial selectins: specialized glycoconjugates that mediate rolling and signaling under flow. Blood. 2011;118(26):6743-6751.

47. Jones SL, Knaus UG, Bokoch GM, Brown EJ. Two signaling mechanisms for activation of $\alpha \mathrm{M} \beta 2$ avidity in polymorphonuclear neutrophils. J Biol Chem. 1998;273(17):10556-10566.

48. Getahun A, Cambier JC. Of ITIMs, ITAMs, and ITAMis: revisiting immunoglobulin Fc receptor signaling. Immunol Rev. 2015;268(1):66-73.

49. Rosales C. Fcy receptor heterogeneity in leu- kocyte functional responses. Front Immunol. 2017;8:280.

50. Greuber EK, Pendergast AM. Abl family kinases regulate Fc $\gamma \mathrm{R}$-mediated phagocytosis in murine macrophages. JImmunol. 2012;189(11):5382-5392.

51. Cui $\mathrm{L}$, et al. c-Abl kinase is required for $\beta 2$ integrin-mediated neutrophil adhesion. JImmunol. 2009;182(5):3233-3242.

52. Futosi K, Németh T, Pick R, Vántus T, Walzog B, Mócsai A. Dasatinib inhibits proinflammatory functions of mature human neutrophils. Blood. 2012;119(21):4981-4991.

53. Futosi K, Mócsai A. Tyrosine kinase signaling pathways in neutrophils. Immunol Rev. 2016;273(1):121-139.

54. Greuber EK, Smith-Pearson P, Wang J, Pendergast AM. Role of ABL family kinases in cancer: from leukaemia to solid tumours. Nat Rev Cancer. 2013;13(8):559-571.

55. Grammer AC, Ryals M, Lipsky PE. A comprehensive approach to identify approved drugs and treatments for repositioning as therapies for systemic lupus erythematosus. Arthritis Res Ther 2014;16(suppl 1):A51.

56. Ward PA, Fattahi F, Bosmann M. New insights into molecular mechanisms of immune complex-induced injury in lung. Front Immunol. 2016;7:86.

57. Banchereau R, et al. Personalized immunomonitoring uncovers molecular networks that stratify lupus patients. Cell. 2016;165(6):1548-1550.

58. Rovin BH, Parikh SV. Lupus nephritis: the evolving role of novel therapeutics. Am J Kidney Dis. 2014;63(4):677-690.

59. Palanichamy A, et al. Neutrophil-mediated IFN activation in the bone marrow alters B cell development in human and murine systemic lupus erythematosus. J Immunol. 2014;192(3):906-918.

60. Sundberg TB, et al. Small-molecule screening identifies inhibition of salt-inducible kinases as a therapeutic strategy to enhance immunoregulatory functions of dendritic cells. Proc Natl Acad Sci U S A. 2014;111(34):12468-12473.

61. Ozanne J, Prescott AR, Clark K. The clinically approved drugs dasatinib and bosutinib induce anti-inflammatory macrophages by inhibiting the salt-inducible kinases. Biochem J. 2015;465(2):271-279.

62. Wölfl M, et al. Src-kinase inhibitors sensitize human cells of myeloid origin to Toll-like-receptor-induced interleukin 12 synthesis. Blood. 2013;122(7):1203-1213.

63. Lamagna C, Hu Y, DeFranco AL, Lowell CA. B cell-specific loss of Lyn kinase leads to autoimmunity. JImmunol. 2014;192(3):919-928.

64. Mustjoki S, et al. Rapid mobilization of cytotoxic lymphocytes induced by dasatinib therapy. Leukemia. 2013;27(4):914-924.

65. Wong S, Witte ON. The BCR-ABL story: bench to bedside and back. Annu Rev Immunol. 2004;22:247-306.

66. Tsuboi, et al. Regulation of human neutrophils Fcg receptor IIA by C5a receptor promotes inflammatory arthritis in mice. Arthritis Rheum. 2011;63(2):467-478.

67. Alcaide P, et al. p120-Catenin prevents neutrophil transmigration independently of RhoA inhibition by impairing Src dependent VE-cad- 
herin phosphorylation. Am J Physiol Cell Physiol. 2012;303(4):C385-C395.

68. Stokol T, et al. C1q governs deposition of circulating immune complexes and leukocyte Fc $\gamma$ receptors mediate subsequent neutrophil recruitment. JExp Med. 2004;200(7):835-846.

69. Chen Y, et al. Fluorescence biomembrane force probe: concurrent quantitation of receptor-ligand kinetics and binding-induced intracellular signal- ing on a single cell. J Vis Exp. 2015;(102):e52975. 70. Evans E, Ritchie K, Merkel R. Sensitive force technique to probe molecular adhesion and structural linkages at biological interfaces. Biophys J.1995;68(6):2580-2587.

71. Chen W, Zarnitsyna VI, Sarangapani KK, Huang J, Zhu C. Measuring receptor-ligand binding kinetics on cell surfaces: from adhesion frequency to thermal fluctuation methods. Cell Mol Bio- eng. 2008;1(4):276-288.

72. Utomo A, et al. Requirement for Vav proteins in post-recruitment neutrophil cytotoxicity in IgG but not complement C3-dependent injury. JImmunol. 2008;180(9):6279-6287.

73. Rosenkranz AR, Knight S, Sethi S, Alexander SI, Cotran RS, Mayadas TN. Regulatory interactions of $\alpha \beta$ and $\gamma \delta \mathrm{T}$ cells in glomerulonephritis. Kidney Int. 2000;58(3):1055-1066. 